PFC/JA-95-10

\title{
Scaling Laws for Two-Dimensional Divertor Modeling
}

\author{
Peter J. Catto, Sergei Krasheninnikov, J. W. Connor* \\ May 1995
}

MIT Plasma Fusion Center

Cambridge, Massachusetts 02139 USA

*UKAEA Government Division, Fusion, Culham, Abindgon, Oxfordshire, OX 14 3DB, UK

This work was supported by the US Department of Energy under contract DE-FG02-91ER-54109. Reproduction, translation, publication, use, and disposal, in whole or in part, by or for the US Government is permitted.

Submitted for publication in: Physics of Plasmas 


\title{
Scaling Laws for Two-Dimensional Divertor Modeling
}

\author{
Peter J. Catto, Sergei Krasheninnikov \\ Massachusetts Institute of Technology \\ Plasma Fusion Center \\ 167 Albany Street, NW16-236 \\ Cambridge, MA 02139 \\ J. W. Connor \\ UKAEA Government Division, Fusion \\ Culthern, Abingdon \\ Oxfordshire, $O X 143 D B, U K$ \\ (Euratom , UKAEA Fusion Association)
}

\begin{abstract}
To gain insight into divertor operation, we employ similarity techniques to investigate whether model systems of equations plus boundary conditions admit scaling transformations that lead to useful divertor similarity scaling laws. These can be used to perform similarity experiments or fully exploit large computer simulations. We adopt fluid plasma models of the divertor region which ignore anomalous processes, and consider neutral descriptions in both the short and long mean free path limits. As usual, the more approximations we make, the more scaling transformations are allowed, leading to fewer independent dimensionless parameters that need to be considered, thereby imposing fewer divertor similarity constraints. The simplest model considered balances electron heat conduction with impurity radiation and places the fewest constraints on divertor similarity. To be able to model detached divertor operation in short mean free path regimes, a fluid neutral description is employed which balances plasma pressure by neutral pressure. In this model the constraints on divertor similarity are most severe. A less constrained long mean free path or Knudsen neutral model is also considered. It models detachment by balancing plasma pressure with momentum transfered to neutrals randomized by collisions with the deep slot sidewalls. The simpler models have relaxed divertor similarity constraints, but all models remain severely restricted by collisionality and parallel heat flux constraints.

PACS numbers: 52.40.Hf, 52.55.Fa
\end{abstract}




\section{Introduction}

Two-dimensional numerical models of divertors employing fluid descriptions of the plasma and either short (fluid) or long (Knudsen) mean free path descriptions of the neutrals contain large numbers of dimensionless parameters that must be varied to investigate all operating regimes expected to be of interest. Given the complexity of the descriptions the task is a rather daunting one. The question arises, therefore, as to whether useful information can be obtained and the number of independent parameters reduced by considering the scaling transformation properties 1.2 of the system of differential equations and boundary conditions. The retention of boundary conditions is a new and necessary feature that must be considered when determining the allowed scaling transformations. If scale transformations can be found for a particular system, then by the invarince principle any quantities evaluated from the same system must satisfy the same scalings. To this end, we consider various divertor models and show that the techniques introduced by Connor and Taylor ${ }^{1}$ and reviewed by Connor ${ }^{2}$ can be employed on boundary conditions as well as the accompanying differential equations to find the constraints on divertor similarity.

The sections that follow consider fluid and Knudsen neutral models with fluid plasma equations and boundary conditions appropriate for complete recycling. Both the short and long mean free path neutral descriptions adopted are capable of modeling the observed drops in temperature, particle flux, and energy flux at the target. The models assume all perpendicular transport is due to the neutrals since they ignore anomalous transport processes. They are best viewed as models of the divertor between the $\mathbf{x}$ point and target. More sophisticated models retaining. anomalous transport are possible only if explicit perpendicular transport models are assumed. In order to avoid such ad hoc assumptions about the anomalous transport coefficients we investigate cases in which they enter only through the scrape off layer width which we assume specified. 
Section II presents the fluid neutral and fluid plasma equations and boundary conditions as obtained from Refs. 3 and 4. The equations and boundary conditions are made dimensionless in Sec.III using the ionization energy of hydrogen, the peak upstream plasma pressure, and an appropriate neutral penetration length. In Sec.IV the scaling transformations and similarity constraints of various fluid descriptions are considered. The use of the technique of Connor and Taylor ${ }^{1,2}$ when boundary conditions must be treated is illustrated in detail in Sec.IV.A for the simple case in which the neutrals are neglected and electron heat conduction balances impurity radiation. The scaling law for the power to the target plates $\mathrm{P}$ divided by the major radius $\mathrm{R}$ is derived and several possible ways to consider similarity are noted. Section IV.B considers a reduced two-dimensional (2-D) fluid neutral model based on the onedimensional (1-D) model shown in Ref.5 to exhibit the key features of divertor detachment. In 2-D we recover Lackner's ${ }^{6} \mathrm{P} / \mathrm{R}=$ constant scaling, while in the 1-D limit P/R need not be held constant for similarity. Moreover, for this fluid neutral model it is found that $P / R \propto \Delta_{p} / \ell_{n}>1$, with $\Delta_{p}$ and $\ell_{n}$ the scrape off layer (SOL) width and neutral penetration length. In Sec.IV.C it is shown that the general 2-D fluid neutral model which does not allow any scaling transformations leads to essentially the same conclusions as the reduced 2-D fluid model, but involves more parameters. Section V describes the Knudsen neutral model and its limitations. It then investigates the scaling transformations for the dimensionless equations and boundary conditions to show that $\mathrm{P} / \mathrm{R} \propto\left(\Delta_{\mathrm{p}} / \ell_{\mathrm{n}}\right)^{3 / 2} \ll 1$ for the Knudsen model and that it need not be held constant for similar devices. In Sec. V we discuss the implications of our results. 


\section{Fluid Neutral and Plasma Equations and Boundary Conditions}

We adopt a simplied version of the full fluid neutral and plasma equations in the SOL as given in Refs. 3 and 4. Most of the simplifications correspond to those normally employed in 2-D SOL codes and are based in part on the 1-D model solved in Ref. 5. Neglecting recombination here and elsewhere, the steady-state ion and neutral continuity equations are

$$
\nabla \cdot\left(\mathbf{N}_{\mathrm{i}} \overrightarrow{\mathrm{V}}_{\mathrm{i}}\right)=\langle\sigma \mathrm{v}\rangle_{\mathrm{z}} \mathbf{N}_{\mathrm{e}} \mathbf{N}_{\mathrm{n}}
$$

and

$$
\nabla \cdot\left(\mathbf{N}_{\mathrm{n}} \overrightarrow{\mathrm{V}}_{\mathrm{n}}\right)=-\langle\sigma \mathrm{v}\rangle_{\mathrm{z}} \mathbf{N}_{\mathrm{e}} \mathbf{N}_{\mathrm{n}},
$$

where the subcripts $e, i$, and $n$ denote electrons, ions, and neutrals; $N_{j}$ and $\vec{V}_{j}$ denote the density and mean velocity of species $\mathrm{j}$; and $\langle\sigma \mathrm{v}\rangle_{\mathrm{z}}$ is the rate constant for electron impact ionization. We assume singly charged ions and employ quasi-neutrality and local ambipolarity to obtain

$$
N_{e}=N_{i} \text { and } \vec{V}_{e}=\vec{V}_{i} \text {. }
$$

We assume that the mean ion velocity perpendicular to the magnetic field $V_{i \perp}$ vanishes,

$$
\overrightarrow{\mathrm{V}}_{\mathrm{i}}=0 \text {, }
$$

and for the perpendicular mean neutral velocity $\ddot{\mathrm{V}}_{\mathrm{n} L}$ we use the perpendicular neutral momentum balance equation to obtain

$$
N_{n} \vec{V}_{n \perp}=\frac{-\nabla_{\perp}\left(N_{n} T\right)+0.24 N_{n} \nabla_{\perp} T}{M N_{i}\langle\sigma v\rangle_{x}+M N_{e}\langle\sigma v\rangle_{z}}
$$

where $\mathrm{M}$ and $\mathrm{T}$ are the mass and temperature of the ions and neutrals and $\langle\sigma v\rangle_{\mathbf{X}}$ is the charge exchange rate constant. The $0.24 \mathrm{~N}_{n} \nabla T$ term is the thermal force found in Ref. 4 and the inertial terms are neglected since we assume that the perpendicular mean flows are small. By taking $\overrightarrow{\mathrm{V}}_{\mathrm{L}}=0$ we are neglecting the anomalous perpendicular particle flux normally retained in the codes. Were explicit expressions available for the anomalous transport coefficients, the boundary conditions could be suitably modified 
and similarity techniques employed on the generalized system of equations. However, divertor simulators and codes need not necessarily model the details of anomalous transport in the SOL. We avoid making ad hoc assumptions about the anomalous transport coefficients in the SOL by specifying the SOL width as an input parameter and by considering only the divertor region between the $\mathrm{x}$-point and the target.

To find the equation for the parallel ion velocity $V_{i \|}$ we add the parallel ion and electron momentum equations to eliminate electron-ion friction and obtain the parallel plasma momentum balance equation

$$
\begin{gathered}
\nabla \cdot\left[\left(\mathrm{MN}_{\mathrm{i}} \mathrm{V}_{\mathrm{i} \mid}^{2}+\mathrm{N}_{\mathrm{i}} \mathrm{T}+\mathrm{N}_{\mathrm{e}} \mathrm{T}_{\mathrm{e}}+\pi_{\mathrm{i}}\right) \hat{\mathbf{n}}\right]=\langle\sigma \mathrm{v}\rangle_{\mathrm{z}} \mathrm{MN}_{\mathrm{e}} \mathrm{N}_{\mathrm{n}} \mathrm{V}_{\mathrm{pl|}} \\
-\langle\sigma v\rangle_{\mathrm{x}} \mathrm{MN}_{\mathrm{n}} \mathrm{N}_{\mathrm{i}}\left(\mathrm{V}_{\mathrm{il}}-\mathrm{V}_{\mathrm{n} \mid}\right)-0.24 \mathrm{~N}_{\mathrm{n}} \hat{\mathrm{n}} \cdot \nabla \mathrm{T},
\end{gathered}
$$

where $\hat{\mathbf{n}}=\mathbf{B} / \mathrm{B}$ is the unit vector along the magnetic field and $T_{e}$ is the electron temperature. Because of charge exchange coupling the parallel ion viscosity $\pi_{i \|}$ is $\left(\mathrm{N}_{\mathbf{n}}+\mathrm{N}_{\mathbf{i}}\right) / \mathrm{N}_{\mathbf{i}}$ times the Braginskii ${ }^{7}$ value 3,4 and therefore given by

$$
\pi_{\mathrm{i} \|}=1.3\left(\mathrm{~N}_{\mathrm{i}}+\mathrm{N}_{\mathrm{n}}\right) \mathrm{T}_{\mathrm{i}} \tau_{\mathrm{i}} \hat{\mathbf{n}} \cdot \nabla \mathrm{V}_{\mathrm{i} \|},
$$

with $\tau_{\mathrm{i}}$ the ion-ion collision time

$$
\tau_{\mathrm{i}}=\frac{3 \mathrm{M}^{1 / 2} \mathrm{~T}^{3 / 2}}{4(\pi)^{1 / 2} \mathrm{~N}_{\mathrm{i}} \mathrm{e}^{4} \ell \mathrm{n} \Lambda} .
$$

The parallel neutral velocity $V_{n \|}$ is found from the parallel neutral momentum equation

$$
\begin{gathered}
\nabla \cdot\left[\left(\mathrm{MN}_{\mathrm{n}} \mathrm{V}_{\mathrm{n}||}^{2}+\mathrm{N}_{\mathrm{n}} \mathrm{T}\right) \hat{\mathbf{n}}++\ddot{\pi}_{\mathrm{n}} \cdot \hat{\mathbf{n}}\right]=-\langle\sigma v\rangle_{\mathrm{z}} \mathrm{MN}_{\mathrm{e}} \mathrm{N}_{\mathrm{n}} \mathrm{V}_{\mathrm{n}||} \\
+\langle\sigma \mathrm{v}\rangle_{\mathrm{x}} \mathrm{MN}_{\mathrm{n}} \mathrm{N}_{\mathrm{i}}\left(\mathrm{V}_{\mathrm{i} \mid}-\mathrm{V}_{\mathrm{n} \mid}\right)+0.24 \mathrm{~N}_{\mathrm{n}} \hat{\mathbf{n}} \cdot \nabla \mathrm{T},
\end{gathered}
$$

where, in the presence of charge exchange coupling to the ions, the neutral viscosity $\pi_{n} \cdot \hat{n}$ is is given by ${ }^{3,4}$

$$
\tilde{\pi}_{\mathbf{n}} \hat{\mathbf{n}}=\left(\frac{\mathbf{N}_{\mathbf{n}}}{\mathbf{N}_{\mathrm{i}}}\right) \pi_{\mathrm{i} \mid \mathbf{n}} \hat{\mathbf{n}}-\frac{\mathbf{N}_{\mathrm{n}} \mathrm{T}}{\mathrm{N}_{\mathrm{i}}\langle\sigma \mathrm{v}\rangle_{\mathrm{x}}}\left[\nabla \mathrm{V}_{\mathrm{n}||}+\frac{1}{3} \hat{\mathbf{n}} \hat{\mathbf{n}} \cdot \nabla \mathrm{V}_{\mathrm{n} \mid}\right] .
$$

Charge exchange causes the ion and neutrals temperatures to be equal to lowest order, as well as coupling the ion and neutral flows via Eq.(6). To close our system of 
equations we need separate equations for the ion/neutral and electron temperatures. Ion plus neutral energy conservation with the viscosity and electric field terms neglected gives the equation for $\mathrm{T}$ to be

$$
\nabla \cdot\left[\left(\frac{5}{2} T+\frac{1}{2} M V_{i}^{2}\right)\left(N_{i}+N_{n}\right) \vec{V}+\vec{q}_{i}+\vec{q}_{n}\right]=\frac{3 m N_{e}\left(T_{e}-T\right)}{M \tau_{e i}},
$$

where $V$ and $V_{\|}$are mean mass velocities defined by

$$
\left(N_{i}+N_{n}\right) \vec{V}=N_{i} \vec{V}_{i}+N_{n} \vec{V}_{n}
$$

$\mathrm{V}^{2} \approx \mathrm{V}_{\|}^{2}$, and $\tau_{\mathrm{ei}}$ is the electron-ion collision time

$$
\tau_{\mathrm{ei}}=\frac{3 \mathrm{~m}^{1 / 2} \mathrm{~T}_{\mathrm{e}}^{3 / 2}}{4(2 \pi)^{1 / 2} \mathrm{~N}_{\mathrm{i}} \mathrm{e}^{4} \ln \Lambda}
$$

Charge exchange makes the parallel ion heat flux $\vec{q}_{i}$ larger than its Braginskii ${ }^{7}$ value by $^{3,4}\left(\mathrm{~N}_{\mathrm{n}}+\mathrm{N}_{\mathrm{i}}\right) / \mathrm{N}_{\mathrm{i}}$ giving

$$
\overrightarrow{\mathrm{q}}_{\mathbf{i}}=-3.9\left[\left(\mathrm{~N}_{\mathrm{i}}+\mathbf{N}_{\mathbf{n}}\right) \mathrm{T} \tau_{\mathbf{i}} / \mathbf{M}\right] \hat{\mathbf{n}} \hat{\mathbf{n}} \cdot \nabla \mathrm{T} .
$$

The neutral heat flux $\vec{q}_{n}$ in the presence of charge exchange coupling to the ions is given by 3,4

$$
\overrightarrow{\mathrm{q}}_{\mathrm{n}}=\left(\frac{\mathrm{N}_{\mathrm{n}}}{\mathrm{N}_{\mathrm{i}}}\right) \overrightarrow{\mathrm{q}}_{\mathrm{i}}-\frac{2.4 \mathrm{~N}_{\mathrm{n}} \mathrm{T}}{\mathrm{MN_{i }}\langle\sigma \mathrm{v}\rangle_{\mathrm{x}}} \nabla \mathrm{T}-0.24 \mathrm{~N}_{\mathrm{n}} \mathrm{T}\left(\overrightarrow{\mathrm{V}}_{\mathrm{n}}-\overrightarrow{\mathrm{V}}_{\mathrm{i}}\right)
$$

where the last term is of the form referred to as a diffusion thermo-effect according to Chapman and Cowling 8 .

The final equation for the electron temperature $T_{e}$ follows from the electron energy conservation equation

$$
\left.\nabla \cdot\left(\frac{5}{2} \mathrm{~T}_{\mathrm{e}} \mathrm{N}_{\mathrm{e}} \overrightarrow{\mathrm{V}}_{\mathrm{e}}+\overrightarrow{\mathrm{q}}_{\mathrm{e}}\right)=-\frac{3 \mathrm{mN} \mathrm{N}_{\mathrm{e}}\left(\mathrm{T}_{\mathrm{e}}-\mathrm{T}\right)}{\mathrm{M} \tau_{\mathrm{ei}}}-(\mathrm{K} \sigma \mathrm{v}\rangle_{\mathrm{z}}+\mathrm{E}_{\mathrm{H}}\langle\sigma \mathrm{v}\rangle_{\mathrm{H}}\right) \mathrm{N}_{\mathrm{e}} \mathrm{N}_{\mathrm{n}}-\mathrm{E}_{\mathrm{I}}\langle\sigma \mathrm{v}\rangle_{\mathrm{I}} \mathrm{N}_{\mathrm{e}} \mathrm{N}_{\mathrm{I}},(
$$

where $\mathrm{I}$ is the ionization potential for hydrogen $(13.6 \mathrm{eV}), \mathrm{E}_{\mathrm{H}}$ is the excitation energy of hydrogen (10.2 eV for Lyman $\alpha$ ) with an excitation rate constant of $\langle\sigma\rangle_{\mathrm{H}}, \mathrm{N}_{\mathrm{I}}$ is the impurity density, and $E_{I}$ is the energy of the relevant excited impurity state with an excitation rate constant of $\langle\sigma v\rangle_{\mathrm{I}}$. The parallel electron heat flux $\overrightarrow{\mathrm{q}}_{e}$ is just the Braginskii $^{7}$ result 


$$
\overrightarrow{\mathrm{q}}_{\mathrm{e}}=-3.2\left(\mathrm{~N}_{\mathrm{e}} \mathrm{T}_{\mathrm{e}} \tau_{\mathrm{ei}} / \mathrm{m}\right) \hat{\mathbf{n}} \hat{\mathbf{n}} \cdot \nabla \mathrm{T}_{\mathrm{e}} .
$$

The preceding system of equations requires ten boundary conditions since it is tenth order in the eight unknowns $N_{e}, N_{n}, V_{i} \|, \nabla_{n}, T e$, and $T$. For upstream boundary conditions we employ

and

$$
\begin{gathered}
\mathrm{N}_{\text {nlup }}=0, \\
\mathrm{~N}_{\mathrm{e}}\left(\mathrm{T}_{\mathrm{e}}+\mathrm{T}\right)_{\mathrm{lup}_{\mathrm{p}}}=\mathrm{P}_{\mathrm{up}}, \\
-3.2\left(\mathrm{~N}_{\mathrm{e}} \mathrm{T}_{\mathrm{e}} \tau_{\mathrm{e}} / \mathrm{m}\right) \hat{\mathbf{n}} \cdot \nabla \mathrm{T}_{\mathrm{dup}}=\mathrm{q}_{\mathrm{ap}}^{\mathrm{up}},
\end{gathered}
$$

$$
-\left.3.9\left[\left(\mathrm{~N}_{\mathbf{i}}+\mathrm{N}_{\mathbf{n}}\right) \mathrm{T} \tau_{\mathrm{i}} / \mathrm{M}\right] \hat{\mathbf{n}} \cdot \nabla \mathrm{T}\right|_{\text {up }}=\mathrm{q}_{\mathrm{i} \|}^{\text {up }},
$$

where the upstream pressure $P_{u p}$, and upstream parallel electron (ion) heat flux $q_{a \|}^{\text {up }}$ $\left(q_{\text {ill }}^{\text {up }}\right)$ are all specified functions at the upstream entrance to the divertor. The neutral density boundary condition corresponds to considering complete recycling in the divertor region since it, ambipolarity, and the sum of the two continuity equations require that there be no plasma entering through the upstream boundary.

The remaining six boundary conditions are applied downstream at the divertor target plates and sidewalls, where wall values of density, temperature, etc. will be denoted by a subscript $w$. The outward directed unit vector normal to the wall is defined as $\hat{\mathbf{w}}$, where for definiteness we assume $\hat{\mathbf{w}} \cdot \hat{\mathbf{n}}>0(\hat{\mathbf{w}} \cdot \hat{\mathbf{n}}<0)$ when $V_{i \|}<0\left(V_{i \|}\right.$ $>0$ ). The parallel ion flow into the walls must satisfy a generalized Bohm sheath criterion,

$$
\hat{\mathbf{w}} \cdot \hat{\mathbf{n}} \mathrm{V}_{\mathbf{i} \|} \|_{\mathbf{w}}=-\hat{\mathbf{w}} \cdot \hat{\mathbf{n}} \alpha\left(\mathrm{T}_{\mathbf{w}} / \mathrm{M}\right)^{1 / 2},
$$

where $\alpha \sim 0.5-1$. To maintain a steady state, complete recycling is imposed at the walls by demanding that the outgoing normal neutral flux equal the incoming normal ion flux,

$$
\hat{\mathbf{w}} \cdot\left(\hat{\mathbf{n}} \mathrm{N}_{\mathrm{i}} \mathrm{V}_{\mathrm{i} \|}+\mathrm{N}_{\mathrm{n}} \nabla_{\mathrm{n}}\right)_{\mathrm{w}}=0 \text {. }
$$

The parallel plasma momentum flux normal to the wall is specified by

$$
\hat{\mathbf{w}} \cdot \hat{\mathbf{n}}\left(\mathrm{MN}_{\mathrm{i}} \mathrm{V}_{i \|}^{2}+\mathrm{N}_{\mathrm{i}} \mathrm{T}+\mathrm{N}_{\mathrm{e}} \mathrm{T}_{\mathrm{e}}+\pi_{\mathbf{i} \|}\right)_{\mathrm{w}}=\gamma_{\|} \hat{\mathbf{w}} \cdot \hat{\mathbf{n}} \mathrm{N}_{\mathrm{iw}} \mathrm{T}_{\mathbf{w}},
$$


where $\gamma_{\|} \sim 2-3$ is the plasma momentum transmission coefficient. Similarly, the normal neutral momentum flux normal to the wall is taken to be

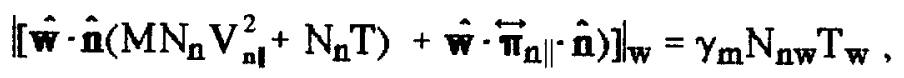

with $\gamma_{\mathbf{m}} \sim 1-2$ the neutral momentum transmission coefficient.

The final wall boundary conditions are on the energy fluxes. The normal ion plus neutral energy flux onto the wall is taken as

$\left.\mid \hat{w} \cdot\left[\left(5 T / 2+M V^{2} / 2\right)\left(N_{n}+N_{i}\right) \vec{V}+\vec{q}_{i}+\vec{q}_{n}\right)\right]\left.\right|_{w}=-\left(\alpha|\hat{w} \cdot \hat{n}| \gamma_{i} N_{i w}+\gamma_{n} N_{n w}\right) T_{w}\left(T_{w} / M\right)^{1 / 2}$

where $\gamma_{i} \sim 2-3$ and $\gamma_{n} \sim 0.1-0.3$ are the ion and neutral heat transmission coefficients, respectively. The $\alpha \dot{\mathbf{w}} \cdot \hat{\mathbf{n}}$ in the ion contribution on the right side accounts for the ion heat flux moving along the magnetic field and ions hitting the wall with a parallel speed satisfying the Bohm condition. A similar expression holds for the normal electron energy flux onto the wall,

$$
\left.\hat{\mathbf{w}} \cdot\left[\hat{\mathbf{n}}\left(5 \mathrm{~T}_{\mathrm{e}} / 2\right) \mathrm{N}_{\mathrm{e}} \mathrm{V}_{\mathrm{el}}+\overrightarrow{\mathrm{q}}_{\mathrm{e}}\right)\right]_{\mathrm{w}}=-\alpha \hat{\mathbf{w}} \cdot \hat{\mathbf{n}} \gamma_{\mathrm{e}} \mathrm{N}_{\mathrm{ew}} \mathrm{T}_{\mathrm{ew}}\left(\mathrm{T}_{\mathrm{ew}} / \mathrm{M}\right)^{1 / 2}
$$

with $\gamma_{\mathrm{e}} \sim 2-3$ the electron heat transmission coefficient. The various transmission coefficients are discussed in Refs. 9.

The preceding equations and boundary conditions form the basic system of equations that we will investigate for fluid neutrals. In the following sections we will make further assumptions that allow us to combine the preceding equations to obtain simpler systems. Moreover, In Sec.V we will make modifications that allow us to consider Knudsen neutrals. 


\section{Dimensionless Form of the Equations and Boundary Conditions}

To determine if the system of equations and boundary conditions (and, therefore, the heat loads on target, etc.) of Sec.II are invariant under particular sets of scaling transformations, it is convenient to first make the entire system dimensionless. To do so, we will adopt a 2-D cartesian model with $\mathrm{x}$ and $\mathrm{y}$ denoting the radial and poloidal coordinates, and $\mathrm{z}$ corresponding to the ignorable toroidal direction $(\partial / \partial \mathrm{z}=0)$. Writing the unit vector along the magnetic field as

$$
\hat{\mathbf{n}}=\left(\mathrm{B}_{\mathrm{T}} / \mathrm{B}\right) \hat{\mathbf{z}}+b \hat{\mathbf{y}},
$$

with $b=B_{P} / B$, and $B_{P}\left(B_{T}\right)$ the poloidal (toroidal) magnetic field components, gives

$$
\hat{\mathbf{n}} \cdot \boldsymbol{\nabla}=b \partial / \partial y, \quad V_{i y}=b V_{i} \|, \quad V_{i z}=\left(B_{T} / B\right) V_{i \|} \quad \text { and } \quad V_{j \|}=b V_{i y}+\left(B_{T} / B\right) V_{i z},(28)
$$

where $j=i, n$ or $e$ and we will assume that $B=|B|$ is a constant.

We normalize temperatures and velocities to the hydrogen ionization potential I by defining

$$
\begin{gathered}
\tau=T / I, \quad \tau_{e}=T_{e} / I, \quad V_{\|}=(M / I)^{1 / 2} V_{i \|}, \\
u=(M / I)^{1 / 2} V_{n y}, \quad w=(M / I)^{1 / 2} V_{n x} \quad \text { and } \quad v=(M / I)^{1 / 2} V_{n \|} .
\end{gathered}
$$

Densities are normalized by introducing the peak upstream plasma pressure $P_{v}$ so that the known function $P_{v p}$ in the upstream pressure boundary condition may be written as

$$
\mathrm{P}_{\mathrm{up}}=\mathrm{P}_{\mathrm{u}} \mathrm{S}_{\mathrm{p}}\left(\mathrm{x} / \Delta_{\mathrm{p}}\right) \text {, }
$$

where $S_{p}\left(x / \Delta_{p}\right)$ is a specified order unity SOL shape function. Using $P_{v}$ and $I$ the normalized plasma and neutral densities are then defined as

$$
\mathrm{n}=\mathbb{I N}_{\mathrm{e}} / \mathrm{P}_{\mathbf{u}}=\mathrm{IN}_{\mathrm{e}} / \mathrm{P}_{\mathbf{u}} \quad \text { and } \quad \boldsymbol{\eta}=\mathbb{N}_{\mathbf{n}} / \mathbf{P}_{\mathbf{v}} .
$$

We desire to normalize lengths to the neutral penetration scale length $\ell_{\mathrm{n}}$ at the temperature $I$ and density $P_{v} / I$. As a result, it is convenient to introduce another set of shape functions $S_{x}(T)$ and $S_{z}\left(T_{e}\right)$ by defining

$$
\langle\sigma v\rangle_{\mathbf{x}}=\mathrm{K}_{\mathbf{x}} \mathrm{S}_{\mathbf{x}}(\mathrm{T}) \text { and }\langle\sigma v\rangle_{\mathrm{z}}=\mathrm{K}_{\mathrm{z}} \mathrm{S}_{\mathrm{z}}\left(\mathrm{T}_{\mathrm{e}}\right) \text {, }
$$


where $\mathrm{K}_{\mathbf{x}}$ and $\mathrm{K}_{\mathbf{z}}$ are true constants equal to the appropriate peak values of the charge exchange and ionization rate constants so that the functions $S_{x}(T)$ and $S_{z}\left(T_{e}\right)$ are of order unity. Using the preceding definitions, $\ell_{\mathrm{n}}$ is defined as

$$
\ell_{\mathrm{n}}=\frac{\mathrm{I}(\mathrm{I} / \mathrm{M})^{1 / 2}}{\mathrm{P}_{\mathrm{u}}\left(\mathrm{K}_{\mathrm{x}} \mathrm{K}_{\mathrm{z}}\right)^{1 / 2}}
$$

and the normalized poloidal $(\beta)$ and radial $(\beta)$ variables may be defined as

$$
\beta=y / \ell_{\mathrm{n}} \text { and } \rho=x / \ell_{\mathrm{n}} .
$$

Using the preceding definitions and introducing the definition

$$
\sigma=\left(\mathrm{K}_{\mathrm{Z}} / \mathrm{K}_{\mathbf{x}}\right)^{1 / 2}
$$

the continuity equations and the perpendicular neutral flux equations become

$$
\begin{gathered}
b \frac{\partial}{\partial \beta}\left(\eta v_{\|}\right)=\sigma n \eta S_{z}(\tau) \\
\frac{\partial}{\partial \beta}(\eta u)+\frac{\partial}{\partial \rho}(\eta w)=-\sigma n \eta S_{z}\left(\tau_{e}\right) \\
\eta(u-b v)=\frac{-\frac{\partial}{\partial \beta}(\eta \tau)+0.24 \eta \frac{\partial \tau}{\partial \beta}}{n\left[\sigma S_{z}\left(\tau_{e}\right)+\sigma^{-1} S_{x}(\tau)\right]}
\end{gathered}
$$

and

$$
\eta w=\frac{-\frac{\partial}{\partial \rho}(\eta \tau)+0.24 \eta \frac{\partial \tau}{\partial \rho}}{n\left[\sigma S_{z}\left(\tau_{e}\right)+\sigma^{-1} S_{x}(\tau)\right]}
$$

To make the parallel plasma momentum equation dimensionless we need to introduce a new dimensionless parameter $\mu$ which is the Coulomb mean free path $\lambda$ divided by the neutral penetration length at temperature I,

$$
\mu=\left.\frac{0.55\left(\mathrm{MK}_{\mathrm{x}} \mathrm{K}_{\mathrm{z}}\right)^{1 / 2} \mathrm{I}^{3 / 2}}{\mathrm{e}^{4} \ell \mathrm{n} \Lambda} \approx \frac{\lambda}{\ell_{\mathrm{n}}}\right|_{\mathrm{T}=\mathrm{I}}
$$

Using this definition in $\pi_{i} \|$, parallel plasma momentum balance becomes

$$
\begin{aligned}
b \frac{\partial}{\partial \beta}\left[n v_{1}^{2}\right. & \left.+n\left(\tau+\tau_{e}\right)+\mu b \tau^{5 / 2} \frac{(n+\eta)}{n} \frac{\partial v_{1}}{\partial \beta}\right]+0.24 b \eta \frac{\partial \tau}{\partial \beta} \\
= & n \eta\left[\sigma v S_{z}\left(\tau_{e}\right)-\sigma^{-1}\left(v_{1}-v\right) S_{x}(\tau)\right]
\end{aligned}
$$


No further new parameters are introduced by neutral momentum balance which upon inserting the neutral viscosity may be written as

$$
\begin{gathered}
\mathrm{b} \frac{\partial}{\partial \beta}\left[\eta v^{2}+\eta \tau+\mu b \tau^{5 / 2} \frac{\eta(n+\eta)}{n^{2}} \frac{\partial v_{\|}}{\partial \beta}\right]-0.24 b \eta \frac{\partial \tau}{\partial \beta}-\frac{\partial}{\partial \beta}\left\lfloor\frac{\sigma\left(1+\frac{1}{3} b^{2}\right) \eta \tau}{n S_{x}(\tau)} \frac{\partial v}{\partial \beta}\right\rceil \\
-\frac{\partial}{\partial \rho}\left\lfloor\frac{\sigma \eta \tau}{n S_{x}(\tau)} \frac{\partial v}{\partial \rho}\right]=-n \eta\left[\sigma v S_{z}\left(\tau_{e}\right)-\sigma^{-1}\left(v_{\|}-v\right) S_{x}(\tau)\right] .
\end{gathered}
$$

To write electron energy balance in dimensionless form it is convenient to first introduce two new shape functions $S_{H}\left(T_{e}\right)$ and $S_{I}\left(T_{e}\right)$ for the hydrogen and impurity radiation by letting

$$
\langle\sigma v\rangle_{H}=K_{H} S_{H}\left(T_{e}\right) \quad \text { and } \quad\left\langle\sigma v>_{I}=K_{I} S_{I}\left(T_{e}\right)\right.
$$

where $K_{H}$ and $K_{I}$ are true constants equal to the appropriate peak values of the hydrogen and impurity rate constants such that the functions $\mathrm{S}_{\mathrm{H}}\left(\mathrm{T}_{\mathrm{e}}\right)$ and $\mathrm{S}_{\mathrm{I}}\left(\mathrm{T}_{\mathrm{e}}\right)$ are order unity functions. Next, we define the dimensionless impurity density $\mathrm{n}_{\mathrm{I}}$ and dimensionless constants $\sigma_{H}$ and $\sigma_{I}$ corresponding respectively to energy and impurity density times energy weighted ratios of radiation to neutral penetration scale lengths,

$$
\mathrm{n}_{\mathrm{I}}=\mathrm{IN}_{\mathrm{I}} / \mathrm{P}_{\mathrm{u}}, \quad \sigma_{\mathrm{H}}=\mathrm{E}_{\mathrm{H}} \mathrm{K}_{\mathrm{H}} / \mathrm{I}\left(\mathrm{K}_{\mathbf{x}} \mathrm{K}_{\mathrm{Z}}\right)^{1 / 2} \text { and } \sigma_{\mathrm{I}}=\mathrm{n}_{\mathrm{I}} \mathrm{E}_{\mathrm{I}} \mathrm{K}_{\mathrm{I}} / \mathrm{I}\left(\mathrm{K}_{\mathbf{x}} \mathrm{K}_{\mathrm{Z}}\right)^{1 / 2}
$$

Notice that we have implicitly assumed that the shape function for $\mathrm{n}_{\mathrm{I}}$ depends only on $\mathrm{T}_{e}$, but we could introduce an additional shape function to remove this restriction. $A$ final dimensionless constant parameter $k$, proportional to $(\mathrm{M} / \mathrm{m})^{1 / 2}$ times the Coulomb mean free path $\lambda$ divided by the neutral penetration length at temperature $I$, is defined by

$$
\mathrm{k}=\left.\frac{0.96 \mathrm{M}\left(\mathrm{K}_{\mathrm{x}} \mathrm{K}_{\mathrm{z}}\right)^{1 / 2} \mathrm{I}^{3 / 2}}{\mathrm{~m}^{1 / 2} \mathrm{e}^{4} \ln \Lambda} \approx\left(\frac{\mathrm{M}}{\mathrm{m}}\right)^{1 / 2} \frac{\lambda}{\ell_{\mathrm{n}}}\right|_{\mathrm{T}_{0}=\mathrm{I}}
$$

Notice that $\mathbf{k}$ is also a measure of the temperature equilibration length divided by the neutral penetration length. In terms of the preceding parameters, the dimensionless electron energy equation becomes

$\mathrm{b} \frac{\partial}{\partial \beta}\left[\frac{5}{2} \tau_{\mathrm{e}} \mathrm{nv_{1 }}-\mathrm{kb} \tau_{\mathrm{e}}^{5 / 2} \frac{\partial \tau_{\mathrm{e}}}{\partial \beta}\right]=-\frac{9.6 \mathrm{n}^{2}\left(\tau_{\mathrm{e}}-\tau\right)}{\mathrm{k \tau _{e } ^ { 3 / 2 }}}-\sigma \mathrm{n \eta} \mathrm{S}_{\mathrm{z}}\left(\tau_{\mathrm{e}}\right)-\sigma_{\mathrm{H}} \mathrm{n \eta} \mathrm{S}_{\mathrm{H}}\left(\tau_{\mathrm{e}}\right)-\sigma_{\mathrm{I}} \mathrm{nS} \mathrm{S}_{\mathrm{I}}\left(\tau_{\mathrm{e}}\right)$ 
The ion plus neutral energy balance introduces no new parameters. With the heat fluxes inserted it becomes the rather complicated expression

$$
\begin{gathered}
\frac{\partial}{\partial \beta}\left\{\left[\frac{5}{2} \tau+\frac{1}{2}\left(\frac{n v_{\|}+\eta v}{n+\eta}\right)^{2}\right\rceil\left(b n v_{1}+\eta u\right)-3 \mu b^{2} \tau^{5 / 2} \frac{(n+\eta)^{2}}{n^{2}} \frac{\partial \tau}{\partial \beta}-0.24 \eta \tau\left(u-b v_{\|}\right)\right\} \\
+\frac{\partial}{\partial \rho}\left\{\left[\frac{5}{2} \tau+\frac{1}{2}\left(\frac{n v_{\|}+\eta v}{n+\eta}\right)^{2}\right\rceil \eta w-0.24 \eta \tau w\right\}-\frac{\partial}{\partial \beta}\left[\frac{2.4 \sigma \eta \tau}{n S_{x}(\tau)} \frac{\partial \tau}{\partial \beta}\right] \\
-\frac{\partial}{\partial \rho}\left\lfloor\frac{2.4 \sigma \eta \tau}{n S_{x}(\tau)} \frac{\partial \tau}{\partial \rho}\right]=\frac{9.6 n^{2}\left(\tau_{c}-\tau\right)}{k \tau_{c}^{3 / 2}},
\end{gathered}
$$

where to simplify this result we have assumed for the first time that Bp (and, therefore, b) is a constant.

To obtain the boundary conditions in dimensionless form it is convenient to introduce the shape functions $S_{\mathrm{e}}\left(\mathrm{x} / \Delta_{\mathrm{p}}\right)$ and $\mathrm{S}_{\mathrm{i}}\left(\mathrm{x} / \Delta_{\mathrm{p}}\right)$ for known upstream poloidal electron $\left(\mathrm{bq}_{\text {*a }}^{\mathrm{up}}\right)$ and ion $\left(\mathrm{bq}_{\mathrm{il|}}^{\mathrm{up}}\right)$ heat fluxes by defining

$$
\mathrm{bq}_{\mathrm{di}}^{\mathrm{up}}=-\mathrm{Q}_{\mathrm{e}} \mathrm{P}_{\mathrm{u}}(\mathrm{I} / \mathrm{M})^{1 / 2} \mathrm{~S}_{\mathrm{e}}\left(\mathrm{x} / \Delta_{\mathrm{p}}\right) \text { and } \quad \mathrm{bq}_{\mathrm{i} \|}^{\mathrm{up}}=-\mathrm{Q}_{\mathrm{i}} \mathrm{P}_{\mathrm{u}}(\mathrm{I} / \mathrm{M})^{1 / 2} \mathrm{~S}_{\mathrm{i}}\left(\mathrm{x} / \Delta_{\mathrm{p}}\right)
$$

The dimensionless parameters $Q_{e}$ and $Q_{i}$ times $P_{u}(I / M)^{1 / 2}$ are the peak upstream values of the poloidal electron and ion heat fluxes, respectively. Letting $y=0$ and $y=L$ denote the target and the location at which the upstream boundary conditions are applied, respectively, and employing the preceding definitions, the dimensionless forms of the upstream boundary conditions for the neutral density, plasma pressure, and electron and ion heat fluxes are as follows:

$$
\begin{gathered}
\eta\left(\beta=L / \ell_{\mathrm{n}}\right)=0 \\
\left.n\left(\tau+\tau_{\mathrm{e}}\right)\right|_{\beta=L / \ell_{\mathrm{n}}}=\mathrm{S}_{\mathrm{p}}\left(\rho \ell_{\mathrm{n}} \mid \Delta_{\mathrm{p}}\right), \\
\left.\mathrm{kb}^{2} \tau_{\mathrm{e}}^{5 / 2} \frac{\partial \tau_{\mathrm{e}}}{\partial \beta}\right|_{\beta=L / \ell_{\mathrm{n}}}=\mathrm{Q}_{\mathrm{e}} \mathrm{S}_{\mathrm{e}}\left(\rho \ell_{\mathrm{n}} / \Delta_{\mathrm{p}}\right)
\end{gathered}
$$

and

$$
\left.3 \mu b^{2} \tau^{5 / 2} \frac{\partial \tau}{\partial \beta}\right|_{\beta=L / \ell_{n}}=Q_{i} S_{i}\left(\rho \ell_{n} / \Delta_{p}\right)
$$


We assume that the magnetic field is parallel to the sidewalls located at $\mathbf{x}= \pm \Delta$ and denote by a subscript $d$ the target plate $(y=0)$. Then, the dimensionless forms of the boundary conditions for the parallel ion flow, parallel plasma momentum, and parallel electron energy flow (which do not involve the sidewalls) follow:

$$
\begin{gathered}
\mathbf{v}_{\| d}=-\alpha \tau_{d}^{1 / 2}, \\
\alpha^{2} n_{d} \tau_{d}+n_{d}\left(\tau_{d}+\tau_{e d}\right)+\left.\mu b \tau_{d}^{5 / 2} \frac{\left(n_{d}+\eta_{d}\right)}{n_{d}} \frac{\partial v_{\| l}}{\partial \beta}\right|_{d}=\gamma_{\| \mid} n_{d} \tau_{d},
\end{gathered}
$$

and

$$
\frac{5}{2} \alpha n_{d} \tau_{e d} \tau_{d}^{1 / 2}+\left.k b \tau_{e d}^{5 / 2} \frac{\partial \tau_{e}}{\partial \beta}\right|_{d}=\alpha \gamma_{e} n_{d} \tau_{e d}^{3 / 2}
$$

The remaining boundary conditions involve the neutrals so we must distinguish between the sidewalls (at which $\hat{\mathbf{w}} \cdot \hat{\mathbf{n}}=\hat{\mathbf{x}} \cdot \hat{\mathbf{n}}=0$ and whose location we denote by subscript s meaning $x= \pm \Delta / 2$ ) and target (at which $\hat{\mathbf{w}} \cdot \hat{\mathbf{n}}=\hat{\mathbf{y}} \cdot \hat{\mathbf{n}}=\mathbf{b}$ ). As a result, for the remaining dimensionless boundary conditions for the ion plus neutral flows, the neutral momentum, and the ion plus neutral energy we find the following forms:

$$
\begin{gathered}
\eta_{d} u_{d}=\alpha b n_{d} \tau_{d}^{1 / 2}, \quad \eta_{s} w_{s}=0 \\
b \eta_{d}\left(v_{d}^{2}+\tau_{d}\right)+\left.\mu b^{2} \tau_{d}^{5 / 2} \frac{\eta_{d}\left(n_{d}+\eta_{d}\right)}{n_{d}^{2}} \frac{\partial v_{l l}}{\partial \beta}\right|_{d}-\left.\frac{\sigma\left(1+\frac{1}{3} b^{2}\right) \eta_{d} \tau_{d}}{n_{d} S_{x}\left(\tau_{d}\right)} \frac{\partial v}{\partial \beta}\right|_{d}=\gamma_{m} \eta_{d} \tau_{d} \\
-\left.\frac{\sigma \eta_{s} \tau_{s}}{n_{s} S_{x}\left(\tau_{s}\right)} \frac{\partial v}{\partial \rho}\right|_{s}=\gamma_{m} \eta_{s} \tau_{s}
\end{gathered}
$$

and

$$
\begin{aligned}
& \left\lceil\frac{2.4 \sigma \eta_{d} \tau_{d}}{n_{d} S_{x}\left(\tau_{d}\right)}+\left.3 \mu b^{2} \tau_{d}^{5 / 2} \frac{\left(n_{d}+\eta_{d}\right)^{2}}{n_{d}^{2}} \sqrt[\partial \tau]{\partial \beta}\right|_{d}+0.24 \eta_{d} \tau_{d}\left(u_{d}-b v_{l d}\right)=\left(\alpha b \gamma_{i} n_{d}+\gamma_{n} \eta_{d}\right) \tau_{d}^{3 / 2}\right. \\
& -\left.\frac{2.4 \sigma \eta_{8} \tau_{s}}{n_{s} S_{x}\left(\tau_{s}\right)} \frac{\partial \tau}{\partial p}\right|_{s}=\gamma_{n} \eta_{s} \tau_{B}^{3 / 2}
\end{aligned}
$$

Our fluid description in its most general form is now complete. In the next section we will consider the properties and simplifications of our system of equations. 


\section{Scaling Transformations and Similarity for Fluid Descriptions}

The full 2-D system of differential equations and boundary conditions as given by Eqs. (36-39), (41), (42), (46), (47), (49-52), (53-55), and (56-58) involves the eight unknowns $n, \eta, v_{1}, w, u, v, \tau$, and $\tau_{\mathrm{e}}$ and the seventeen dimensionless parameters $\sigma$, b, $\mu, k, \sigma_{H}, \sigma_{I}, \alpha, Q_{e}, Q_{i}, \gamma_{1}, \gamma_{m}, \gamma_{e}, \gamma_{i}, \gamma_{n}, L / \ell_{n}, \Delta_{\mathrm{p}} / \ell_{n}$, and $\Delta / \ell_{n}$. It is important to notice that $P_{v}$ only appears in the definitions $n, \eta, \ell_{n}, \sigma_{I}$, and through the normalizations for the upstream heat fluxes as given by Eq. (48). As a result, for similar configurations the densities (recall $\sigma_{I} \propto n_{I} \propto N_{I} / P_{v}$ ) must scale linearly with $P_{u}$, while the depth (L) and width $\left(\Delta_{p}\right.$ and $\Delta$ ) scaling must be inversely proportional to $P_{v}$. Recall also that $Q_{e} \alpha$ $\mathrm{bq}_{\mathrm{el}}^{\mathrm{up}} / \mathrm{P}_{\mathrm{u}}$ because of the normalization of the upstream heat fluxes. Notice that the usual collisionality scaling of density times scale length is embedded in $L / \ell_{n}, \Delta_{p} / \ell_{n}$, and $\Delta / \ell_{\mathrm{n}}$ which are proportional to density through $\mathrm{P}_{\mathrm{v}}$.

Fortunately, most of the dimensionless parameters are reasonably well known, but rather wide ranges of $\mathrm{b}, \sigma_{\mathrm{I}}, \mathrm{Q}_{\mathrm{e}}, \mathrm{Q}_{\mathrm{i}}, \mathrm{L} / \ell_{\mathrm{n}}, \Delta_{\mathrm{p}} / \ell_{\mathrm{n}}$, and $\Delta / \ell_{\mathrm{n}}$ are of interest since they depend on the upstream plasma pressure and heat fluxes, the impurities, and the divertor configuration. Typically $\sigma \sim 0.1-1, \sigma_{1} \sim 1$, and for a tokamak $b \sim 0.05$; while for $\mathrm{P}_{\mathrm{v}} \sim 10^{14} \mathrm{~cm}^{-3} \times 100 \mathrm{eV}, \lambda \sim \ell_{\mathrm{n}} \sim 0.3 \mathrm{~cm}$, giving $\mu \sim 1$ and $\mathrm{k} \sim 50$.

To reduce the number of dimensionless parameters we can seek scale transformations of this system of equations. It will turn out that the full system does not allow any scale transformations, but that by simplifying the system, scale transformations can be found which allow us to reduce the number of dimensionless parameters that need be considered.

\section{A. Model Without Neutrals}

We first employ the Connor and Taylor ${ }^{1,2}$ procedure on a simple limit to illustrate the need to retain boundary conditions and how they are easily included by their technique. If we adopt the simplest model possible by neglecting the neutrals completely ( $\eta \rightarrow 0$ ), letting $v_{\|} \rightarrow 0$ and $\tau=\tau_{e}$, adding the energy equations to eliminate 
electron-ion energy exchange, and neglecting the ion heat conduction compared to that of the electrons, we find that plasma pressure balance becomes $2 n \tau_{e}=S_{p}\left(\rho \ell_{n} / \Delta_{p}\right)$, so that we need only consider

$$
2 b \frac{\partial}{\partial \beta}\left[k b \tau_{e}^{5 / 2} \frac{\partial \tau_{c}}{\partial \beta}\right]=\sigma_{I} \tau_{e}^{-1} S_{I}\left(\tau_{e}\right) S_{p}\left(\rho \ell_{n} \mid \Delta_{p}\right) .
$$

In this limit the upstream boundary condition is given by Eq. (51), and the target boundary condition is given by Eq.(55) with the convection term neglected,

$$
\left.2 \mathrm{~kb} \tau_{\text {ed }}^{5 / 2} \frac{\partial \tau_{\mathrm{e}}}{\partial \beta}\right|_{d}=\alpha \gamma_{\mathrm{e}} \tau_{\mathrm{ed}}^{1 / 2} \mathrm{~S}_{\mathrm{p}}\left(\rho \ell_{\mathrm{n}} / \Delta_{\mathrm{p}}\right)
$$

In this model the parameter $\Delta / \ell_{\mathrm{n}}$ does not enter because sidewall boundary conditions are not needed since $\Delta_{\mathrm{p}}<\Delta$

We seek scale transformations by scaling the dependent variable $\tau_{e}$, the two independent variables $\rho$ and $\beta$, and the seven dimensionless parameters $b, k, \sigma_{I}, Q_{e}$, $\alpha \gamma_{e}, L / \ell_{n}$, and $\Delta_{p}$ (but only the arguments of shape functions since their coefficients are accounted for by scaling the dimensionless parameters). Letting $\tau_{e} \rightarrow \omega_{1} \tau_{e}, \beta \rightarrow \omega_{2} \beta$, $\rho \rightarrow \omega_{3} \rho, b \rightarrow \omega_{4} b, k \rightarrow \omega_{5} k, \sigma_{I} \rightarrow \omega_{6} \sigma_{I}, Q_{e} \rightarrow \omega_{7} Q_{e}, \alpha \gamma_{e} \rightarrow \omega_{8} \alpha \gamma_{e}, L / \ell_{n} \rightarrow \omega_{9} L / \ell_{n}$, and $\Delta_{\mathrm{p}} / \ell_{\mathrm{n}} \rightarrow \omega_{10} \Delta_{\mathrm{p}} / \ell_{\mathrm{n}}$ we find four independent scale transformations:

(i) $\beta \rightarrow \omega_{2} \beta, L / \ell_{n} \rightarrow \omega_{2} L / \ell_{n}, k \rightarrow \omega_{2} k, \sigma_{I} \rightarrow \sigma_{I} / \omega_{2}$;

(ii) $\rho \rightarrow \omega_{3} \rho, \Delta_{\mathrm{p}} / \ell_{\mathrm{n}} \rightarrow \omega_{3} \Delta_{\mathrm{p}} / \ell_{\mathrm{n}}$;

(iii) $b \rightarrow \omega_{4} b, \sigma_{I} \rightarrow \omega_{4} \sigma_{I}, k \rightarrow k / \omega_{4}, Q_{e} \rightarrow \omega_{4} Q_{e}$; and

(iv) $Q_{e} \rightarrow \omega_{7} Q_{e}, \sigma_{I} \rightarrow \omega_{7} \sigma_{I}, k \rightarrow \omega_{7} k, \alpha \gamma_{e} \rightarrow \omega_{7} \alpha \gamma_{e}$;

where $\omega_{j}$ denotes the scaling constants and for each transformation we do not indicate quantities not scaled (notice that $\tau_{e}$ can never be scaled because of the complicated dependence of $\mathrm{S}_{\mathrm{I}}$ ). The second transformation is needed to keep the argument of $\rho$ dependent shape functions fixed.

We started with seven dimensionless parameters and two dimensionless independent variables and have found four transformations. Any physical quantity of 
interest derivable from this system of equations must be invariant under the four scaling transformations. In particular, we can find the five invariant combinations of the seven dimensionless parameters and the two dimensionless variables by considing the scaling properties of the invariant product

$$
Z_{A B C . . .}=b^{A} k^{B} \sigma_{I}^{C} Q_{e}^{D}\left(\alpha \gamma_{e}\right)^{E}\left(L / \ell_{n}\right)^{F}\left(\Delta_{p} / \ell_{n}\right)^{G} \rho^{H} \beta^{I}
$$

For both sides of Eq.(62) to be invariant $\left(Z_{\mathrm{ABC}} \ldots \rightarrow \mathrm{Z}_{\mathrm{ABC}} \ldots\right)$ under the scale transformations of $\mathrm{Eq} .(61)$ requires

or

$$
1=\omega_{2}^{\mathrm{B}-\mathrm{C}+\mathrm{F}+\mathrm{I}} \omega_{3}^{\mathrm{G}+\mathrm{H}} \omega_{4}^{\mathrm{A}-\mathrm{B}+\mathrm{C}+\mathrm{D}} \omega_{7}^{\mathrm{B}+\mathrm{C}+\mathrm{D}+\mathrm{E}}
$$

$$
\mathrm{F}=\mathrm{C}-\mathrm{B}-\mathrm{I}, \mathrm{G}=-\mathrm{H}, \mathrm{A}=\mathrm{B}-\mathrm{C}-\mathrm{D} \text {, and } \mathrm{E}=-\mathrm{B}-\mathrm{C}-\mathrm{D} \text {, }
$$

which when used in Eq.(62) gives

$$
Z_{\mathrm{BCDHI}}=\left(\mathrm{bk} \ell_{\mathrm{n}} / \mathrm{L} \alpha \gamma_{\mathrm{e}}\right)^{\mathrm{B}}\left(\sigma_{\mathrm{I}} \mathrm{L} / \ell_{\mathrm{n}} \mathrm{b} \alpha \gamma_{\mathrm{e}}\right)^{\mathrm{C}}\left(\mathrm{Q}_{\mathrm{e}} / \mathrm{b} \alpha \gamma_{\mathrm{e}}\right)^{\mathrm{D}}\left(\rho \ell_{\mathrm{n}} / \Delta_{\mathrm{p}}\right)^{\mathrm{H}}\left(\beta \ell_{\mathrm{n}} / \mathrm{L}\right)^{\mathrm{I}}
$$

As a result, only the three independent dimensionless parameters $b k \ell_{n} / L \alpha \gamma_{e}$, $\sigma_{\mathrm{I}} \mathrm{L} / \ell_{\mathrm{n}} \mathrm{b} \alpha \gamma_{\mathrm{e}}$, and $\mathrm{Q}_{\mathrm{e}} / \mathrm{b} \alpha \gamma_{\mathrm{e}}$, as well as the independent variable combinations $\rho \ell_{\mathrm{n}} / \Delta_{\mathrm{p}}$ and $\beta \ell_{\mathrm{n}} / \mathrm{L}$ satisfy the four scaling transformations, reducing the number of dimensionless quantities by four.

We are particularly interested in the form of the normalized poloidal energy flux on the target plates $Q_{t} \equiv q_{t} / P_{v}(L / M)^{1 / 2}$, which depends on $\rho \ell_{n} / \Delta_{p}$ but not $\beta \ell_{n} / L$ since $\beta=0$. We first note that $Q_{t}$ must contain a coefficient having the same scaling as $Q_{e}$ $\left(Q_{t} \rightarrow w_{4} \omega_{7} Q_{t}\right)$ times an unknown function of these three independent parameters and $\rho \ell_{\mathbf{n}} / \Delta_{\mathbf{p}}$

$$
\mathrm{q}_{\mathrm{t}}=\mathrm{Q}_{\mathrm{e}} \mathrm{P}_{\mathrm{u}}(\mathrm{I} / \mathrm{M})^{1 / 2} \mathrm{f}\left(\mathrm{bk} \ell_{\mathrm{n}} / L \alpha \gamma_{\mathrm{e}}, \sigma_{\mathrm{I}} \mathrm{L} / \ell_{\mathrm{n}} \mathrm{b} \alpha \gamma_{\mathrm{e}}, \mathrm{Q}_{\mathrm{e}} / \mathrm{b} \alpha \gamma_{\mathrm{e}}, \rho \ell_{\mathrm{n}} / \Delta_{\mathrm{p}}\right)
$$

Here and elsewhere $f$ is used to denote an unknown function of the arguments listed. The arguments of $f$ can be written in various equivalent ways since products of the invariant dimensionless quantities are invariant. A convenient form for our purposes is 


$$
q_{t}=b \alpha \gamma_{e} P_{u}(I / M)^{1 / 2} f\left(b k \ell_{n} / L \alpha \gamma_{e}, k \sigma_{I} / \alpha^{2} \gamma_{e}^{2}, Q_{e} / b \alpha \gamma_{e}, \rho \ell_{n} / \Delta_{p}\right)
$$

where $Q_{e}$ and bor $\gamma_{e}$ scale the same way.

Since the hydrogen ionization potential does not enter this simple model we can make the replacement $I \rightarrow E_{I}$ in all quantities to replace $I$ by the excitation energy of interest. Notice that none of the dimensionless parameters that enter Eqs. (64) or (65) depend on $\mathrm{K}_{\mathbf{X}} \mathrm{K}_{\mathbf{z}}$ since the neutral penetration length, which no longer enters, is replaced by $b$ times an appropriate Coulomb mean free path $\left(0.96 \mathrm{E}_{\mathrm{I}}^{3} / \mathrm{P}_{\mathrm{u}} \mathrm{e}^{4} \ell \mathrm{n} \Lambda\right)$. In this simple model, these same dimensionless parameters can be found by direct integration and application of the boundary conditions. Then, it can be seen that for a sufficiently localized shape function $S_{I}\left(\tau_{e}\right)$ with a $\tau_{e}$ dependence permitting no impurity radiation losses for $y>L$, the function $f$ in Eq. (65) will be insensitive to the first parameter which describes the Coulomb collisionality. Moreover, for a sufficiently low impurity density, f will be insensitive to the second parameter.

If we form the power to the plates $P$ by integrating $x$ over the SOL width $\Delta_{p}$, then for a single null divertor $P=4 \pi R \int d x q_{t}$ gives

$$
\mathrm{P} / \mathrm{R}=\mathrm{b} \alpha \gamma_{\mathrm{e}} \Delta_{\mathrm{p}} \mathrm{P}_{\mathrm{u}}(\mathrm{I} / \mathrm{M})^{1 / 2} \mathrm{f}\left(\mathrm{bk} \ell_{\mathrm{n}} / \mathrm{L} \alpha \gamma_{\mathrm{e}}, \mathrm{k} \sigma_{\mathrm{I}} / \alpha^{2} \gamma_{\mathrm{e}}^{2}, \mathrm{Q} \mathrm{e} / \mathrm{b} \alpha \gamma_{\mathrm{e}}\right)
$$

where $R$ is the major radius of the tokamak and, of course, $f$ is a different unknown function. Notice that the only dependence on the SOL width enters as an explicit multiplier in Eq.(66). For similar divertors we must keep the unknown function $f$ fixed. Since $b k \ell_{n} / L \alpha \gamma_{e} \propto b / \alpha \gamma_{e} L P_{v}$, we may use $P_{v} \propto b / \alpha \gamma_{e} L$ in Eq. (66) to obtain

$$
\mathrm{P} / \mathrm{R} \propto b \propto \gamma_{\mathrm{e}} \mathrm{P} u \Delta_{\mathrm{p}} \propto \mathrm{b}^{2} \Delta_{\mathrm{p}} / L
$$

for similar devices in which bk $\ell_{\mathrm{n}} / \mathrm{L} \alpha \gamma_{\mathrm{e}}, \mathrm{k} \sigma_{\mathrm{I}} / \alpha^{2} \gamma_{\mathrm{e}}^{2}$, and $\mathrm{Q}_{\mathrm{e}} / \mathrm{b} \alpha \gamma_{\mathrm{e}}$ are held constant to keep the unknown function $f$ constant. If $f$ is independent of its first argument (localized $\mathrm{S}_{\mathrm{I}}$ ) then the final form of Eq.(67) is not relevant. 
In Lackner's ${ }^{6}$ treatment all lengths and magnetic fields in the divertor must scale the same way for similar tokamaks or similar divertors so that $\Delta_{\mathrm{p}} / \mathrm{L}$ and $\mathrm{b}$, and, therefore, $P / R$ are constants. It should be mentioned that Lackner actually takes $P$ to be the power from the core that crosses the separatrix, rather than the power entering the divertor. In our restricted model $\Delta_{p}, P_{u}, b, Q_{e}$, and $k \sigma_{I} \propto n_{I} E_{I} K_{I}$, are viewed as independent control parameters ( $\mathrm{k}$ is a constant and $\alpha \gamma_{\mathrm{e}}$ does not vary significantly), so for a given $L$ we can adjust $P_{v}, k \sigma_{I} \propto n_{I} E_{I} K_{I}$, and $Q_{e}$ to keep the arguments of $f$ fixed and still be free to adjust $\Delta_{p}$ and $b$. The constraints and $P / R$ scaling for this model are summarized in the first column of Table I.

Similarity constraints are significantly relaxed by considering this substantially reduced description with no neutrals. In ITER ${ }^{10} \mathrm{P} \sim 300 \mathrm{MW}, \mathrm{R} \sim 8 \mathrm{~m}, \mathrm{~b} \sim 1 / 6, \Delta_{\mathrm{p}} \sim 1 \mathrm{~cm}$, and $P_{v} \sim 1014 \mathrm{~cm}^{-3} \times 500 \mathrm{eV}$, so using Eq.(67), a similar device with $P \sim 30 \mathrm{MW}$ and $R \sim 4 \mathrm{~m}$ gives $P_{I T E R} / R_{T E R} \sim 5 P / R$ and is possible for a $P_{u}$ about five times smaller (but $L$ five times larger) than the ITER value, assuming roughly the same $b, \Delta_{p}$, and $\alpha \gamma_{e}$ (if $\Delta_{p}$ in ITER turns out to be larger than $1 \mathrm{~cm}$ then the power of the similar device could be lowered by the same amount). If we employ the anticipated Alcator C-MOD numbers $\left(P \sim 8 \mathrm{MW}, \mathrm{R} \sim 0.7 \mathrm{~m}, \Delta_{\mathrm{p}} \sim 1 \mathrm{~cm}\right.$, and $\left.\mathrm{P}_{\mathrm{u}} \sim 10^{14} \mathrm{~cm}^{-3} \times 100 \mathrm{eV}\right)$, we get a value of $\mathrm{P} / \mathrm{RP}_{\mathrm{u}} \Delta_{\mathrm{p}}$ larger than the ITER value by almost a factor of two so that it could provide good similarity to ITER in the fluid neutral limit if the collisionality constraint $\left(b \mathbf{b k} \ell_{\mathbf{n}} / \mathrm{L} \alpha \gamma_{\mathrm{e}}{ }^{\alpha}\right.$ $b / \alpha \gamma_{\mathrm{e}} \mathrm{L} P_{\mathfrak{u}}=$ constant) did not need to be satisfied because of sufficiently localized impurity radiation losses such as those that may be occuring when divertor detachment is observed ${ }^{11-14}$.

In addition to $\Delta_{p}$ and $b$, we are also free to adjust $B$ (since only the ratio $b$ enters in our equations). These three adjustments can be used to satisfy other constraints, such as gyroradius over scale length (for example, constant $B \Delta_{p}$ or $B_{p} \Delta_{p}$ ) and/or plasma beta (constant $\beta \propto \mathrm{P}_{\mathrm{v}} / \mathrm{B}^{2}$ ) constraints. Some possibilities are shown in Table II, and include $a P / R \propto b$ scaling similar to that found In Ref. 15 . 


\section{B. Reduced Fluid Neutral Model}

If we assume that $\sigma<<1$ and $b<<1$, and neglect inertial $\left(v^{2} \approx v_{\|}^{2}<<\tau \sim 1\right)$ and ion $\left(\sigma \mu \mathrm{b}^{2}<<1\right)$ and neutral viscosity $(\sigma<<1)$ effects, then the ionization terms in Eqs. (38) and (39) may be neglected and Eq. (41) or (42) employed to find $v \approx v_{\| 1}$. As a result, the perpendicular neutral flux equations, Eqs. (38) and (39), become

$$
\mathrm{n} \eta\left(\mathrm{u}-\mathrm{bv}_{1}\right) \mathrm{S}_{\mathrm{x}}(\tau)=\sigma\left[-\frac{\partial}{\partial \beta}(\eta \tau)+0.24 \eta \frac{\partial \tau}{\partial \beta}\right]
$$

and

$$
\mathrm{n} \eta \mathrm{SS}_{\mathrm{x}}(\tau)=\sigma\left[-\frac{\partial}{\partial \rho}(\eta \tau)+0.24 \eta \frac{\partial \tau}{\partial \rho}\right]
$$

If we also assume that the ions and neutrals have equilibrated with the electrons, the sum of Eqs.(41) and (42) with inertial and viscous terms neglected gives total parallel momentum balance to be

$$
\frac{\partial}{\partial \beta}[(2 n+\eta) \tau]=0 .
$$

Equilibration between electrons and ions and neutrals follows from Eq.(47) if convection and conduction are made small by taking $k \sigma<<1$ since we have already assumed perpendicular flows to be small compared to parallel flows and $b<<1$. We obtain the equation for $\tau$ under the same assumptions, by adding Eqs.(46) and (47) and using $\mathrm{k} \gg>\mu$ to form the total energy conservation equation

$$
\begin{aligned}
& \mathrm{b} \frac{\partial}{\partial \beta}\left(5 \tau n v_{1}+0.24 \tau \eta v_{\|}-k b \tau^{5 / 2} \frac{\partial \tau}{\partial \beta}\right)+\frac{\partial}{\partial \beta}(2.3 \tau \eta u)+\frac{\partial}{\partial \rho}(2.3 \tau \eta w) \\
& -\frac{\partial}{\partial \beta}\left[\frac{2.4 \sigma \eta \tau}{n S_{x}(\tau)} \frac{\partial \tau}{\partial \beta}\right\rceil-\frac{\partial}{\partial \rho}\left\lfloor\frac{2.4 \sigma \eta \tau}{n S_{x}(\tau)} \frac{\partial \tau}{\partial \rho}\right]=-\sigma n \eta S_{z}(\tau)-\sigma_{H} n \eta S_{H}(\tau)-\sigma_{I} n S_{I}(\tau)
\end{aligned}
$$

Equations (36), (37), and (68)-(71) are the reduced fluid neutral equations which consist of a fifth order system of six equations for the six unknowns $n, \eta, v_{\|}, u$, w, and

$\tau$. For the orderings $\partial / \partial \beta \sim 1 \sim \partial / \partial \rho, n \sim 1 \sim \eta, v_{\|}^{2}<<\tau \sim 1, \sigma<<1, b<<1, k \sigma<<1$, and $\mu \sim 1$, 
we see that $b v_{\|} \sim u \sim w \sim k b^{2} \sim \sigma_{H} \sim \sigma_{I}<<1$. The 1-D version of this reduced system of equations with the thermal force and impurity radiation neglected is investigated in Ref.5. To complete the reduced fluid neutral description we need the five boundary conditions given by the Bohm sheath criterion, Eq.(53); complete recycling, Eq.(56); the upstream pressure and heat flux,

$$
\begin{gathered}
\left.2 \mathrm{n} \tau\right|_{\beta=\mathrm{L} / \ell_{\mathrm{n}}}=\mathrm{S}_{\mathrm{p}}\left(\rho \ell_{\mathrm{n}} \mid \Delta_{\mathrm{p}}\right), \\
\left.\mathrm{kb}^{2} \tau^{5 / 2} \frac{\partial \tau}{\partial \beta}\right|_{\beta=\mathrm{L} / \ell_{\mathrm{n}}}=\mathrm{Q}_{\mathrm{e}} \mathrm{S}_{\mathrm{e}}\left(\rho \ell_{\mathrm{n}} \mid \Delta_{\mathrm{p}}\right) ;
\end{gathered}
$$

and the energy flux into the walls,

$$
\begin{gathered}
\frac{5}{2} \alpha b n_{d} \tau_{d}^{3 / 2}+\left.\left[k^{2} \tau_{d}^{5 / 2}+\frac{2.4 \sigma \eta_{d} \tau_{d}}{n_{d} S_{x}\left(\tau_{d}\right)}\right] \frac{\partial \tau}{\partial \beta}\right|_{d}+0.24 \eta_{d} \tau_{d}\left(u_{d}-b v_{j \mid d}\right) \\
=\alpha b\left(\gamma_{e}+\gamma_{i}\right) n_{d} \tau_{d}^{3 / 2}+\gamma_{n} \eta_{d} \tau_{d}^{3 / 2} \\
-\left.\frac{2.4 \sigma \eta_{g} \tau_{s} \partial \tau}{n_{s} S_{x}\left(\tau_{s}\right)} \frac{\partial \tau}{\partial \rho}\right|_{s}=\gamma_{n} \eta_{s} \tau_{s}^{3 / 2}
\end{gathered}
$$

Notice that Eqs.(72) and (73) follow from (50) and (51), while Eq.(74) follows from (55) and (58). Equation (49) is no longer needed since it follows from integrating (70) and using (72), and Eqs.(54) and (57) are not needed because of the neglect of viscous effects.

Equations (36), (37), (68)-(71), (53), (56), and (72)-(74) permit only two independent scale transformations:

$$
\begin{aligned}
& \text { (i) } \sigma \rightarrow \omega_{1} \sigma, b \rightarrow \omega_{1} b, u \rightarrow \omega_{1} u, w \rightarrow \omega_{1} w, k \rightarrow k / \omega_{1}, \sigma_{H} \rightarrow \omega_{1} \sigma_{H}, \sigma_{I} \rightarrow \omega_{1} \sigma_{I}, \\
& Q_{e} \rightarrow \omega_{1} Q_{e}, \gamma_{n} \rightarrow \omega_{1} \gamma_{n} ; \quad \text { and } \\
& \text { (ii) } v_{\|} \rightarrow \omega_{2} v_{\|}, b \rightarrow b / \omega_{2}, k \rightarrow \omega_{2}^{2} k, \alpha \rightarrow \omega_{2} \alpha .
\end{aligned}
$$

In this case the poloidal energy flux onto the plates, $q_{t}$, depends on $\rho$ and the twelve parameters $b, k, \sigma, Q_{e}, \ell_{n} / L, \sigma_{H}, \sigma_{\mathrm{I}}, \alpha, \gamma_{n}, \gamma_{e}+\gamma_{i}, \Delta_{\mathrm{p}} / \ell_{\mathrm{n}}$ and $\Delta / \ell_{\mathrm{n}}$. The parameter 
$\Delta / \ell_{\mathrm{n}}$ enters because of the sidewall boundary condition of Eq.(74b). Recalling that $\mathrm{q}_{\mathrm{t}}$ scales as $Q_{e}$ and proceding as before, gives

$$
\mathrm{q}_{\mathrm{t}}=\mathrm{b} \alpha \mathrm{P}_{\mathrm{u}}(\mathrm{I} / \mathrm{M})^{1 / 2} \mathrm{f}\left(\ell_{\mathrm{n}} / \mathrm{L}, \mathrm{bk} / \alpha, \mathrm{Q}_{\mathrm{e}} / \mathrm{b} \alpha, \sigma_{\mathrm{I}} / \sigma, \sigma / \mathrm{b} \alpha, \sigma_{\mathrm{H}} / \sigma, \gamma_{\mathrm{n}} / \mathrm{b} \alpha, \gamma_{\mathrm{e}}+\gamma_{\mathrm{i}}, \Delta / \mathrm{L}, \Delta / \Delta_{\mathrm{p}}, \rho \ell_{\mathrm{n}} \mid \Delta_{\mathrm{p}}\right)
$$

The dimensionless parameters that enter $q_{t}$ are consistent (apart from notation) with those found in the one-dimensional model of Ref.5 which neglects thermal force effects (and modifications required by Onsager symmetry), impurity radiation in its recycling region, and the parameters $\ell_{\mathrm{n}} / \mathrm{L}, \Delta / \mathrm{L}$, and $\Delta / \Delta_{\mathrm{p}}$ that depend on the depth and width of the divertor channel and scrape off layer to find

$$
\tau_{\mathrm{d}}=\mathrm{f}\left(\mathrm{b}^{2} \mathrm{k} / \sigma, \mathrm{Q}_{\mathrm{e}} / \mathrm{b} \alpha, \sigma / \mathrm{b} \alpha, \sigma_{\mathrm{H}} / \sigma, \gamma_{\mathrm{n}} / \mathrm{b} \alpha, \gamma_{\mathrm{e}}+\gamma_{\mathrm{i}}\right),
$$

which is then solved to obtain the following form of the global energy balance equation:

$$
\mathrm{Q}_{\mathrm{e}}=\mathrm{b} \alpha \mathrm{f}\left(\tau_{\mathrm{d}}, \mathrm{b}^{2} \mathrm{k} / \sigma, \sigma / \mathrm{b} \alpha, \sigma_{\mathrm{H}} / \sigma, \gamma_{\mathrm{n}} \mathrm{lb} \alpha, \gamma_{\mathrm{e}}+\gamma_{\mathrm{i}}\right) \text {. }
$$

Forming the power to the plates $P$ for a single null divertor using $P=4 \pi R \int d x q_{t}$ to integrate Eq. (76) over the scrape off layer width $\Delta_{p}$ gives

$$
\mathrm{P} / \mathrm{R}=\mathrm{b} \alpha \Delta_{\mathrm{p}} \mathrm{P}_{\mathrm{u}}(\mathrm{I} / \mathrm{M})^{1 / 2} \mathrm{f}\left(\ell_{\mathrm{n}} / \mathrm{L}, \mathrm{bk} / \alpha, \mathrm{Q}_{\mathrm{e}} / \mathrm{b} \alpha, \sigma_{\mathrm{I}} / \sigma, \sigma / \mathrm{b} \alpha, \sigma_{\mathrm{H}} / \sigma, \gamma_{\mathrm{n}} / \mathrm{b} \alpha, \gamma_{\mathrm{e}}+\gamma_{\mathrm{i}}, \Delta / \mathrm{L}, \Delta_{\mathrm{p}} / \Delta\right)
$$

For similar devices described by this reduced fluid neutral model we need to keep $f$ fixed by keeping each of the dimensionless parameters constant. To see to what extent this is possible we note that the parameters $\mathrm{k}, \sigma, \sigma_{\mathrm{H}}$, and $\ell_{\mathrm{n}} \mathrm{P}_{\mathrm{v}}$ are constants and that $\alpha, \gamma_{\mathfrak{n}} / \alpha$, and $\gamma_{\mathrm{e}}+\gamma_{\mathrm{i}}$ do not vary significantly. For a specified depth $\mathrm{L}$ we adjust $P_{\mathfrak{v}}$, $\mathrm{b}, \mathrm{Q}_{\mathrm{e}}$, and $\sigma_{\mathrm{I}}$ to hold $\ell_{\mathrm{n}} / \mathrm{L} \propto 1 / \mathrm{LP} \mathrm{P}_{\mathrm{v}}, \mathrm{bk} / \alpha, \mathrm{Q}_{\mathrm{e}} \mathrm{b} \alpha$, and $\sigma_{\mathrm{I}} / \sigma$ fixed, then we must adjust $\Delta$ and $\Delta_{\mathrm{p}}$ to keep $\Delta / \mathrm{L}$ and $\Delta / \Delta_{\mathrm{p}}$ constant. Therefore, we must keep

$$
\mathrm{P} / \mathrm{R} \propto \mathrm{b} \alpha \Delta_{\mathrm{p}} \mathrm{P}_{\mathrm{v}} \propto \Delta_{\mathrm{p}} / \ell_{\mathrm{n}}=\text { constant }
$$

Consequently, similarity for this fluid neutral model recovers Lackner's $^{6}$ result and requires the same $b, Q_{e}\left(\right.$ recall that $\left.Q_{e} \propto q_{e \| l}^{\mathrm{up}} / P_{u}\right), \ell_{n} / L \propto 1 / L P_{v}, \sigma_{I}, \Delta / L$, and $\Delta / \Delta_{p}$, as 
well as the same $\alpha, k, \sigma, \sigma_{H}, \gamma_{n}$, and $\gamma_{\mathrm{e}^{+}} \gamma_{\mathrm{i}}$. For the fluid neutral model, B (or $\mathrm{B}_{\mathrm{p}}$ ) is the only adjustable quantity that can be used to satisfy either a gyroradius or beta scaling. The second column of Table I summarizes the constraints and $P / R$ scaling (which, of course, can be re-expressed using the constraints) for this model.

It is important to note that the constant $\Delta_{\mathrm{p}} / \ell_{\mathrm{n}}$ must be much larger than unity for the fluid neutral model since the SOL thickness must be small compared to the neutral penetration scale length. As a result, the larger $P / R$, the more the neutrals behave like a fluid. Except for Alcator-C-MOD ${ }^{11}$, current machines ${ }^{12-14}$ are in the opposite limit of Knudsen neutrals. However, there is recent experimental evidence from Alcator-C-MOD that the detached divertor operation observed is insensitive to the depth ${ }^{16} \mathrm{~L}$. If this observation is confirmed it would mean that $\mathrm{f}$ is insensitive to $\mathrm{L}$ in detached regimes (as in the 1-D model of Ref.5), then the parameters $\ell_{n} / L$ and $\Delta / L$ in $f$ could be replaced by the single $\mathrm{L}$ independent parameter $\Delta / \ell_{\mathrm{n}} \propto \alpha \Delta_{\mathrm{p}} / \ell_{\mathrm{n}}$. Recall that in the model without neutrals the insensitivity to $L$ occurred whenever the radiation losses were sufficiently localized.

We can relax the P/R scaling of this neutral fluid model by considering the onedimensional limit of Eqs.(36), (37), (68)-(71), (53), (56), and (72)-(74) in which $\partial / \partial \rho=0$ $=w$ in Eqs. (37), (69), and (71) for the SOL region $\left.|\rho|<\Delta_{\mathrm{p}} / 2 \ell_{\mathrm{n}} \quad|\mathrm{x}|<\Delta_{\mathrm{p}} / 2\right)$ and the sidewalls and the sidewall boundary condition (74b) do not enter. In this case, which includes the model of Ref.5, $\Delta$ cannot enter $q_{t}$ and $P / R$. Moreover, the scaling transformation (ii) of Eq.(61) is allowed since $\rho$ enters only through shape functions, which means that $\Delta_{p}$ does not enter the unknown function in $P / R$ and only enters $q_{t}$ via the combination $\rho \ell_{\mathrm{n}} / \Delta_{\mathrm{p}}$. In addition, the divertor is asssumed infinitely deep $(\mathrm{L} \rightarrow \infty)$ and impurity radiation is assumed to occur upstream so that $L$ and $\sigma_{I}$ do not enter. As a result, for the one-dimensional neutral fluid case

$$
\mathrm{qt}_{\mathrm{t}}=\mathrm{b} \alpha \mathrm{Pu}_{\mathrm{u}}(\mathrm{I} / \mathrm{M})^{1 / 2} \mathrm{f}\left(\mathrm{bk} / \alpha, \mathrm{Q}_{\mathrm{e}} / \mathrm{b} \alpha, \sigma / \mathrm{b} \alpha, \sigma_{\mathrm{H}} / \sigma, \gamma_{\mathrm{n}} / \mathrm{b} \alpha, \gamma_{\mathrm{e}}+\gamma_{\mathrm{i}}, \rho \ell_{\mathrm{n}} / \Delta_{\mathrm{p}}\right)
$$




$$
\mathrm{P} / \mathrm{R}=\mathrm{b} \alpha \Delta_{\mathrm{p}} \mathrm{Pu}(\mathrm{I} / \mathrm{M})^{1 / 2} \mathrm{f}\left(\mathrm{bk} / \alpha, \mathrm{Q}_{\mathrm{e}} / \mathrm{b} \alpha, \sigma / \mathrm{b} \alpha, \sigma_{\mathrm{H}} / \sigma, \gamma_{\mathrm{n}} / \mathrm{b} \alpha, \gamma_{\mathrm{e}}+\gamma_{\mathrm{i}}\right)
$$

and, upon choosing $b$ and $Q_{e}$ to hold the unknown function $f$ fixed (recall $k, \sigma, \sigma_{H}$, and $\ell_{\mathbf{n}} \mathrm{P}_{\mathrm{v}}$ are constants, and $\alpha$ and the $\gamma$ 's do not vary significantly),

$$
\mathrm{P} / \mathrm{R} \propto \Delta_{\mathrm{p}} \mathrm{P}_{\mathrm{u}} \propto \Delta_{\mathrm{p}} / \ell_{\mathrm{n}} .
$$

Unlike the 2-D case, $\Delta_{\mathrm{p}} / \ell_{\mathrm{n}}$ need not be a constant for similar devices in the 1-D case. Therefore, a 1-D model of a SOL of width $\Delta_{p}$ allows a device similar to ITER to have a much smaller $P / R$ and $\Delta_{p} P_{v} \propto \Delta_{p} / \ell_{n}$ as in the discussion following Eq. (67).

If we include the inertial terms in our reduced neutral fluid model then we lose the second scaling transformation in Eq.(75) since $v_{\|}^{2}$ and $\tau$ must scale in the same way. As a result, $\alpha$ must be held constant for similarity since it appears as a separate parameter in the argument of $\mathrm{f}$. Since $\alpha \approx$ constant to satisfy the Bohm sheath criterion, the modified $\mathrm{f}$ is effectively the same as Eq. (78) and, therefore, leads to the same conclusions.

If, in addition, we keep ion heat conduction and neutral and ion viscosity, but assume $b^{2}<<1$, we need only supplement the first scaling transformation in Eq.(75) by $\mu \rightarrow \mu / \omega_{1}, \gamma_{m} \rightarrow \omega_{1} \gamma_{m}$ and $Q_{i} \rightarrow \omega_{1} Q_{i}$. Then the only allowed scaling transformation gives Eq. (78) with the additional dimensionless parameters $\alpha, b \mu, \gamma_{m} / b, \gamma_{1}$ and $Q_{i} / b$ appearing in the argument of the unknown function. Since $\mu$ is a constant and $\gamma_{m}$ and $\gamma_{\|}$ do not vary significantly, and $b$ had to be held constant to keep $f$ fixed (note that $b k$ is one of the arguments of $f$ and $k$ is a constant), viscosity and ion heat conduction only alter our conclusions from Eqs. (76) and (78) by requiring that each of the dimensionless upstream electron and ion heat fluxes, $Q_{e}$ and $Q_{i}$, respectively, be held fixed in similar devices.

Finally, if we attempt to keep the ionization terms as well as the charge exchange terms in the perpendicular neutral momentum balance equations, (38) and (39), we will also lose the first scaling transformation in Eq. (75) because $\sigma \rightarrow \sigma$. Then 
the unknown function in $\mathrm{q}_{t}$ will depend on the sixteen dimensionless parameters $\sigma, b$, $\mu, \mathbf{k}, \sigma_{H}, \sigma_{I}, \alpha, Q_{e}, Q_{i}, \gamma_{1}, \gamma_{m}, \gamma_{e}+\gamma_{i}, \gamma_{n}, \Delta / L, \Delta / \Delta_{p}$, and $L / \ell_{n}$, as well as $\rho \ell_{n} / \Delta_{p}$. Since $\sigma, \mu, k, \sigma_{H}, \alpha, \gamma_{l}, \gamma_{m}, \gamma_{e} \gamma_{i}$, and $\gamma_{n}$ are either constants or unable to vary significantly, the additional ionization terms have no significant impact on similarity since only the same $b, \sigma_{I}, Q_{e}, Q_{i}, \Delta / L, \Delta \Delta_{p}$, and $L / \ell_{n}$ are required.

\section{General Fluid Neutral Mode1}

The general fluid neutral model system of equations described in Sec.III cannot allow any scaling transformations since none are permitted for the reduced system mentioned at the end of Sec.IV.B. Neglecting ion and neutral viscosity, inertial terms, and ionization in the perpendicular neutral flow equations does not help because of the neutral-ion momentum exchange due to charge exchange and the ion-electron energy equilibration terms. Consequently, for the general neutral fluid system no simplification occurs so

$$
P / R=\Delta_{p} P_{u}(I / M)^{1 / 2} f\left(\sigma, b, \mu, k, \sigma_{H}, \sigma_{I}, \alpha, Q_{e}, Q_{i}, \gamma_{1}, \gamma_{m}, \gamma_{e}, \gamma_{i}, \gamma_{n}, \Delta / L, \Delta / \Delta_{p}, L / \ell_{n}\right),
$$

with $Q_{e}$ and $Q_{i}$ and $\gamma_{e}$ and $\gamma_{i}$ appearing separately rather than as sums. In this case similarity requires the same $b, \sigma_{I}, \Delta / L, \Delta / \Delta_{p}, L / \ell_{n} \propto P_{\mathbf{u}} L, Q_{e}$, and $Q_{i}$ (with $\sigma, \mu, k, \sigma_{H}$, $\alpha, \gamma_{1}, \gamma_{m}, \gamma_{e}, \gamma_{i}$, and $\gamma_{n}$ either constants or unable to vary significantly), and, not surprisingly, leads to Lackner's $8 / R=$ constant result. Therefore, the general fluid neutral model introduces additional parameters, but leads to essentially the same conclusions as the reduced fluid neutral model.

\section{Scaling Transformations and Similarity for Knudsen Neutrals}

Reference 5 also considers a deep divertor slot geometry with a complete recycling model of the neutrals in which the neutral mean free path is long compared to the divertor width and the Coulomb mean free path is assumed small compared to parallel scale lengths. This idealized limit, in which charge exchange is retained and the 
long mean free path neutrals are randomized by collisions with the walls, is referred to as the Knudsen neutral model or Knudsen flow approximation in Ref.5. For this model the depth of the narrow divertor slot $\mathrm{L}$ must be much larger than its width $\Delta$ so that nearly all of the neutrals created at the target by the recombining ions can stream to the sidewalls to be randomized within a few $\Delta$ 's of the target and well before they reach the upstream divertor entrance. As a result, the neutrals are assumed to be uniformly distributed in $\mathbf{x}$ (as well as $\mathrm{z}$ ) for $\Delta \ll \mathrm{y}<\mathrm{L}$ with no poloidal or toroidal flow. The randomizing wall collisions are assumed to result in a diffusive poloidal neutral flow

$$
N_{n} \vec{V}_{n}=-\hat{y} D \partial N_{n} / \partial y
$$

where the diffusion coefficient $\mathrm{D}$ is given by

$$
\mathrm{D}=\mathrm{cV} \Delta
$$

with $\Delta$ and $V$ the characteristic step in $\mathrm{y}$ and speed between randomizing collisions at the walls and $c$ an order unity numerical coefficient which depends on the properties and conditioning of the walls. The speed $V$ is set by the neutral temperature which in this model is small compared to the ion and electron temperatures. Equation (82) is then inserted into Eq.(2) to obtain the neutral continuity equation. To make D dimensionless we introduce the dimensionless diffusivity d defined by

$$
\mathrm{d}=\frac{\mathrm{cV} \Delta}{\ell_{\mathrm{n}}(\mathrm{I} / \mathrm{M})^{1 / 2}}
$$

The remaining equations for the Knudsen neutral model are ion continuity, Eq.(1) with Eq.(4) inserted; parallel plasma momentum balance as obtained from Eq.(6) by setting $\mathrm{V}_{\mathrm{n} \mid}=0$, neglecting inertia, and dropping the thermal force term; and total plasma energy conservation as obtained by assuming $\mathrm{T}_{\mathrm{e}} \approx \mathrm{T}$, neglecting inertia, and adding Eqs.(11) and (16) together with the neutral flow and neutral heat flux terms ignored. If we neglect ion heat conduction and viscosity the dimensionless form of the Knudsen neutral model equations is as follows: 


$$
\begin{gathered}
d \frac{\partial^{2} \eta}{\partial \beta^{2}}=\sigma n \eta S_{z}(\tau), \\
b \frac{\partial}{\partial \beta}\left(n v_{1}\right)=\sigma n \eta S_{z}(\tau), \\
\sigma b \frac{\partial}{\partial \beta}(2 n \tau)=-n \eta v_{1} S_{x}(\tau), \\
b \frac{\partial}{\partial \beta}\left(5 \tau n v_{1}-k b \tau^{5 / 2} \frac{\partial \tau}{\partial \beta}\right)=-\sigma n \eta S_{z}(\tau)-\sigma_{H} n \eta S_{H}(\tau)-\sigma_{I} n S_{I}(\tau) .
\end{gathered}
$$

This sixth order system of equations in the four unknowns $n, \eta, v_{\|}$, and $\tau$ is employed with the three upstream boundary conditions given by Eqs. (49), (72), and (73), and the three downstream boundary conditions given by Eq. (53) and the appropriately modified versions of (56) and (74a):

$$
\begin{gathered}
-\left.\mathrm{d} \frac{\partial \eta}{\partial \beta}\right|_{d} \Delta=\alpha b \int_{d}^{\Delta_{p}} \mathrm{dx} n_{d} \tau_{d}^{1 / 2}=\alpha b \Delta_{p} \int_{d}^{1} d\left(\rho \ell_{\mathrm{n}} \mid \Delta_{\mathrm{p}}\right) \mathrm{n}_{\mathrm{d}} \tau_{\mathrm{d}}^{1 / 2}, \\
5 \alpha b n_{d} \tau_{d}^{3 / 2}+\left.\mathrm{kb}^{2} \tau_{d}^{5 / 2} \frac{\partial \tau}{\partial \beta}\right|_{\mathrm{d}}=\alpha \mathrm{b}\left(\gamma_{\mathrm{c}}+\gamma_{\mathrm{i}}\right) \mathrm{n}_{\mathrm{d}} \tau_{\mathrm{d}}^{3 / 2} .
\end{gathered}
$$

The three downstream boundary conditions are applied a few $\Delta$ 's from the wall in onder for the diffusive model of Eqs. (82) to be valid. Equation (87) is obtained by demanding complete recycling, and recalling that the neutral distribution is uniform across the entire divertor channel of width $\Delta$, while the plasma is localized to the SOL of width $\Delta_{p}$. Notice that $\Delta$ enters through $d$, as well as Eq.(87), but not through any sidewall boundary conditions, and recall that $\beta=\mathrm{L} / \ell_{\mathrm{n}}$ at the upstream entrance.

The Knudsen neutral model, consisting of Eqs.(49), (53), (72), (73), and (83)(88), contains the twelve dimensionless parameters $\mathrm{d}, \sigma, \mathrm{b}, \mathrm{k}, \sigma_{\mathrm{H}}, \sigma_{\mathrm{I}}, \mathrm{L} / \ell_{\mathrm{n}}, \mathrm{Q}_{\mathrm{e}}, \alpha$, $\gamma_{\mathrm{e}}+\gamma_{\mathrm{i}}, \Delta_{\mathrm{p}} / \ell_{\mathrm{n}}$, and $\Delta_{\mathrm{p}} / \Delta$, and permits the following three scale transformations:

(i) $\sigma \rightarrow \omega_{1} \sigma, v_{\|} \rightarrow \omega_{1} v_{\|}, k \rightarrow \omega_{1} k, \sigma_{H} \rightarrow \omega_{1} \sigma_{H}, \sigma_{I} \rightarrow \omega_{1} \sigma_{I}, d \rightarrow \omega_{1} d$ $\mathrm{Q}_{\mathrm{e}} \rightarrow \omega_{1} \mathrm{Q}_{\mathrm{e}}, \alpha \rightarrow \omega_{1} \alpha$;

(ii) $b \rightarrow \omega_{2} b, \beta \rightarrow \omega_{2} \beta, d \rightarrow \omega_{2}^{2} d, Q_{e} \rightarrow \omega_{2} Q_{e}, L / \ell_{n} \rightarrow \omega_{2} L / \ell_{n} ;$ and

(iii) $\rho \rightarrow \omega_{3} \rho, \Delta_{\mathrm{p}} / \ell_{\mathrm{n}} \rightarrow \omega_{3} \Delta_{\mathrm{p}} / \ell_{\mathrm{n}}$. 
These transformations reduce the number of independent dimensionless parameters by three and the procedure of Sec.IV.A gives

$$
\begin{aligned}
& \mathrm{q}_{\mathrm{t}}=\mathrm{b} \alpha \mathrm{P}_{\mathrm{u}}(\mathrm{I} / \mathrm{M})^{1 / 2} \mathrm{f}\left(\mathrm{b} \ell_{\mathrm{n}} / \mathrm{L}, \mathrm{k} / \alpha, \mathrm{Q}_{\mathrm{e}} / \mathrm{b} \alpha, \sigma_{\mathrm{I}} / \sigma, \sigma / \alpha, \sigma_{\mathrm{H}} / \sigma, \mathrm{d} / \alpha \mathrm{b}^{2}, \gamma_{\mathrm{e}}+\gamma_{\mathrm{i}}, \Delta_{\mathrm{p}} / \Delta, \rho \ell_{\mathrm{n}} / \Delta_{\mathrm{p}}\right) \\
& \text { and } \\
& \mathrm{P} / \mathrm{R}=\mathrm{b} \alpha \Delta_{\mathrm{p}} \mathrm{P}_{\mathrm{u}}(\mathrm{I} / \mathrm{M})^{1 / 2} \mathrm{f}\left(\mathrm{b} \ell_{\mathrm{n}} / \mathrm{L}, \mathrm{k} / \alpha, \mathrm{Q}_{\mathrm{e}} / \mathrm{b} \alpha, \sigma_{\mathrm{I}} / \sigma, \sigma / \alpha, \sigma_{\mathrm{H}} / \sigma, \mathrm{d} / \alpha \mathrm{b}^{2}, \gamma_{\mathrm{e}}+\gamma_{\mathrm{i}}, \Delta_{\mathrm{p}} / \Delta\right)
\end{aligned}
$$

The new parameter in the unknown function is the effective diffusivity $d / \alpha b^{2}$ (or $\ell_{n} d / \alpha b L \propto c V \Delta / a b L$ ) which replaces the $\gamma_{n} / b \alpha$ (or $\gamma_{n} \Delta / b \alpha L$ ) parameter of the fluid neutral model $\left(\mathrm{cV}\right.$ replaces $\left.\gamma_{n}\right)$. Moreover, for the deep slot Knudsen model $\Delta / \mathrm{L}$ no longer enters and the $b$ dependence is altered ( $\beta$ scales the same way as $b$ ) from the fluid neutral model since the neutrals are no longer strongly coupled to the ions by charge exchange. The other parameters $\sigma / \alpha, \sigma_{\mathrm{H}} / \sigma, \mathrm{k} / \alpha$, and $\gamma_{\mathrm{e}} \gamma_{\mathrm{i}}$ in the argument of $\mathrm{f}$ for $P / R$ must also be held constant. Therefore, if we adjust $P_{v}, \Delta, \Delta_{p}, Q_{e}$, and $\sigma_{I}$ to keep $b \ell_{\mathrm{n}} / \mathrm{L}, \mathrm{d} / \alpha \mathrm{b}^{2} \propto\left(\mathrm{cV} \Delta \mathrm{P}_{\mathrm{v}} / \alpha \mathrm{b}^{2}\right), \Delta_{\mathrm{p}} / \Delta, \mathrm{Q}_{\mathrm{e}} / \mathrm{b} \alpha$, and $\sigma_{\mathrm{I}} / \alpha$ fixed for a specified $\mathrm{L}$, we obtain the scaling

$$
\mathrm{P} / \mathrm{R} \propto \mathrm{b} \alpha \Delta_{\mathrm{p}} \mathrm{P}_{\mathrm{u}} \propto \mathrm{b}^{2} \Delta_{\mathrm{p}} / \mathrm{L} \propto(\mathrm{cV})^{2}\left(\Delta_{\mathrm{p}} / \mathrm{L}\right)^{3} \propto(\mathrm{cV})^{1 / 2}\left(\Delta_{\mathrm{p}} / \ell_{\mathrm{n}}\right)^{3 / 2}
$$

where we have used $\mathrm{P}_{\mathrm{v}} \propto 1 / \ell_{\mathrm{n}} \propto \mathrm{b} / \mathrm{L}$ and $\mathrm{b} \propto\left(\mathrm{cV} \Delta_{\mathrm{p}} / \alpha \ell_{\mathrm{n}}\right)^{1 / 2}$, and $\alpha$ must be kept constant since $\sigma$ and $\mathrm{k}$ are constants. As a result, $\mathrm{P} / \mathrm{R}$ is not a constant for the Knudsen fluid model, which requires $\Delta_{\mathrm{p}} / \ell_{\mathrm{n}} \ll 1$, and we are still free to adjust $\mathrm{cV}$ and $\mathrm{b}$, as well as $\mathrm{B}$ to satisfy other constraints. The constraints and P/R scaling for the Knudsen model are summarized in the third column of Table I. Keeping the inertial corrections in the Knudsen neutral model results in the loss of scaling transformation (ii) and makes it necessary to keep $\alpha$ fixed for similarity, so results in no significant change.

\section{Discussion}

Based on the model without neutrals and the fluid neutral models considered here, the collisionality, upstream parallel heat flux, and $P / R$ constraints on ITER divertor similarity are all difficult to satisfy in present tokamaks. The model without 
neutrals has the least restrictive $P / R$ scaling and the fluid neutral model the most constraints. The less restrictive and more favorable scaling of the Knudsen model means that the constant $P / R$ constraint found for fluid neutrals is relaxed as the neutrals make the transition from $\Delta_{\mathrm{p}} / \ell_{\mathrm{n}} \gg 1$ to $\Delta_{\mathrm{p}} / \ell_{\mathrm{n}} \ll 1$, where $\Delta_{\mathrm{p}}$ and $\ell_{\mathrm{n}}$ are the scrape off layer width and the characteristic neutral penetration depth [recall Eq.(33)], respectively. Consequently, lower density machines may have a less constrained $P / R$ scaling than higher density ones. In either limit, however, severe similarity constraints arise from the upstream parallel heat flux and collisionality. For a given $b=B_{p} / B$, similar collisionality requires $\mathrm{P}_{\mathrm{v}} \mathrm{L}=$ constant and therefore deep divertor chambers at the lower upstream pressures of present tokamaks. However, in light of recent experimental results from Alcator-C-MOD 16 indicating that divertor operation during detachment is insensitive to the divertor depth $L$, it is tempting to speculate that it may be possible to ignore the $L$ dependence of $f$ in Eq.(91) and replace the $\ell_{n} / L$ and $\Delta / L$ dependences of the unknown function $f$ in Eq. (78) by $\Delta / \ell_{n}$. Even if this is not the case, the data base from present machines coupled with the key parameters found here and in divertor modeling codes, might be used to construct a power law form for the unknown function in the $P / R$ scaling law. The techniques employed herein can be used to determine the key parameters for the equations and boundary conditions solved in the modeling codes of interest.

The upstream heat flux entering the divertor also places a severe constraint on the similarity of present tokamak divertors to ITER. However, for the various models considered only the parallel heat flux must be matched. Since a divertor simulator need not be a conventional tokamak the parallel heat flux might be matched by adjusting the field line angle in a toroidal device to make $b=B_{p} / B$ smaller for a fixed poloidal heat flux. For a non-conventional tokamak simulator there is much more flexibility in making the collisionality and other divertor and geometrical parameters similar (for a simulator of SOL length $\&$ the replacement $R \rightarrow £ / 4 \pi$ is made in $P / R$ ). 


\section{Acknowledgments}

P. J. C. is pleased to acknowledge the hospitality and support of the Theoretical and Strategic Studies Department, UKAEA Government Division, Fusion, at Culham Laboratory, where much of this work was performed.

This research was jointly supported by U. S. Department of Energy Grant No. DE-FG02-91ER-54109 at the Plasma Fusion Center of the Massachusetts Institute of Technology and the United Kingdom Department of Trade and Industry and Euratom. 


\section{References}

1. J. W. Connor and J. B. Taylor, Nucl. Fusion 1 7, 1047 (1977).

2. J. W. Connor, Plasma Phys. and Controlled Fusion 2 6, 1419 (1984).

3. P. J. Catto, Phys. Plasmas 1, 1936 (1994).

4. P. Helander, S. I. Krasheninnikov, and P. J. Catto, Phys. Plasmas 1, 3174 (1994).

5. S. I. Krasheninnikov, P. J. Catto, P. Helander, D. J. Sigmar, and T. K. Soboleva, "Thermal Bifurcation of Scrape Off Layer Plasma and Divertor Detachment", to be published in Phys. Plasmas.

6. K. Lackner, Comments Plasma Phys. Controlled Fusion 1 5, 359 (1994).

7. S. I. Braginskii, Zh. Exp. Teor. Fiz. 33, 459 (1957) [Sov. Phys. JETP 6, 358 (1958)]; and Reviews of Plasma Physics (Consultants Bureau, New York, 1965), Vol. 1, p. 205.

8. S. Chapman and T. G. Cowling, The Mathematical Theory of Non-Uniform Gases, 3rd ed. (Cambridge University Press, Cambridge, 1970).

9. K. Lackner, R. Chodura, M. Kaufmann, J. Neuhauser, K. G. Rauh, and W. Schneider, Plasma Phys. Control. Fusion 26105 (1984); and Yu. L. Igikhanov, S.I. Krasheninnikov, A.S. Kukushkin, and P.N. Yushmanov, in Reviers of Science and Technology, Plasma Physios, edited by V.D. Shafranov (USSR Inst of Scientific and Technological Information, Moscow, 1990), Vol. 11, p. 5 (in Russian).

10. G. Janeschitz, in Proceedings of the 15th Lnternational Conference on Plasma Physics and Controlled Nuclear Fusion Research, Seville, Spain, September, 1994 (International Atomic Energy Agency, Vienna, 1994), Paper No. IAEA-CN-60/EP-6-1.

11. I.H. Hutchinson, R. Boivin, F. Bombarda, P. Bonoli, S. Fairfax, C. Fiore, J. Goetz, S. Golovato, R. Granetz, M. Greenwald, S. Horne, A. Hubbard, J. Irby, 
B. LaBombard, B. Lipschultz, E. Marmar, G. McCracken, M. Porkolab, J.

Rice, J. Snipes, Y. Takase, J. Terry, S. Wolfe, C. Christensen, D. Garnier, M.

Graf, T. Hsu, T. Luke, M. May, A. Nemczewski, G. Tinios, J. Schachter, and J. Urban, Physics of Plasmas 1, 1511 (1994).

12. T. W. Petrie, D. Buchenauer, D.N. Hill, C. Klepper, S. Allen, R. Campbell, A. Futch, R. J. Groebner, A, Leonard, S. Lippmann, M. Ali Mahdavi, M. Rensink, and P. West, Proceedings of the 10th International Conference on PlasmaSurface Interaction on Controlled Fusion Devices, Monterey, CA, USA, March 30-April 3, 1992, edited by W. Bauer, W. L. Hsu, G. L. Jackson, G. D. Porter, Journal of Nucl. Materials 196-198 (North-Holland, Amsterdam, 1992), p. 848.

13. G. Janeschitz, S. Clement, N. Gottardi, M. Lesourd, J. Lingertat, C. Lowry, G Radford, G. Saibene, M. Stamp, D. Summers, A. Taroni, P. R. Thomas, and G. Vlases, Proceedings of 19th European Conference on Controlled Fusion and Plasma Physios, Insbruck, 1992 (European Physical Society, Petit-Lancy, Switzerland, 1992), Vol. 16C, Part II, 727.

14. V. Mertens, K. Buchl, W. Junker, F. Mast, M. Schittenhelm, M. BessenrodtWeberpals, A. Field, Ch. Fuchs, O. Gehre, O. Gruber, A. Herrmann, G. Haas, A. Kallenbach, H. Kastelewicz, M. Kaufmann, W. Koppendorfer, M. Laux, G. Lieder, J. Neuhauser, F. Ryter, H. Salzmann, W. Sandmann, K.-H. Steurer, A. Stabler, H. Zohm, and Asdex Upgrade Team, Proceedings of 20th European Conference on Controlled Fusion and Plasma Physics, Lisboa, 1993 (European Physical Society, Petit-Lancy, Switzerland, 1993), Vol. 17C, Part I, 267.

15. I. H. Hutchinson and G. C. Vlases (private communication, Aug.1994).

16. Alcator C-MOD divertor group (private communication, Feb.1995). 
Table 1. Divertor Similarity: Constraints (key arguments of $f$ ) and $P / R$ Scaling (coefficient of $f$ )

\begin{tabular}{|c|c|c|c|}
\hline $\begin{array}{c}\text { Similarity } \\
\text { Constraint } \\
\end{array}$ & $\begin{array}{c}\text { No Neutrals } \\
\text { (3 arguments) }\end{array}$ & $\begin{array}{c}\text { Fluid Neutrals } \\
\text { (6 key arguments) }\end{array}$ & $\begin{array}{l}\text { Knudsen Neutrals } \\
\text { (5 key arguments) }\end{array}$ \\
\hline $\begin{array}{l}\text { parallel heat flux } \\
\text { (determines } \mathrm{Q}_{\mathrm{e}} \text { ) }\end{array}$ & $\begin{array}{c}Q_{e} / b \alpha \gamma_{e} \\
\left(\alpha \gamma_{e} \approx \text { constant }\right)\end{array}$ & $Q_{\mathrm{e}} / \mathrm{b}$ & $\mathrm{Q}^{\prime} \mathrm{b}$ \\
\hline $\begin{array}{l}\text { impurity radiation } \\
\left.\text { (determines } \sigma_{1}\right)\end{array}$ & $\begin{array}{c}\sigma_{\mathrm{I} /\left(\alpha \gamma_{\mathrm{e}}\right)^{2}} \\
\left(\alpha \gamma_{\mathrm{e}} \approx \text { constant }\right)\end{array}$ & $\sigma_{\mathrm{I}}$ & $\sigma_{I}$ \\
\hline $\begin{array}{c}\text { collisionality } \\
\text { (determines L) }\end{array}$ & $\begin{array}{c}\mathrm{b} \ell_{\mathrm{n}} / \alpha \gamma_{\mathrm{e}} \mathrm{L} \\
\left(\alpha \gamma_{\mathrm{e}} \approx \text { constant }\right)\end{array}$ & $\mathrm{b} \ell_{\mathrm{n}} / \mathrm{L}$ & $\mathrm{b} \ell_{\mathrm{n}} / \mathrm{L}$ \\
\hline $\begin{array}{l}\text { chamber width } \\
\text { (determines } \Delta \text { ) }\end{array}$ & none & $\Delta / L$ & $\begin{array}{c}d / b^{2} \\
\left(d \propto c V \Delta \vee \ell_{n}\right)\end{array}$ \\
\hline $\begin{array}{c}\text { B field ratio } \\
\text { (determines b) }\end{array}$ & none & b & none \\
\hline $\begin{array}{c}\text { SOL width } \\
\text { (determines } \Delta_{\mathrm{p}} \text { ) }\end{array}$ & none & $\Delta_{\mathrm{p}} / \Delta$ & $\Delta_{\mathrm{p}} / \Delta$ \\
\hline other constants & $\mathbf{k}$ & $\mathrm{k}, \sigma, \alpha, \sigma_{\mathrm{H}}, \gamma_{\mathrm{n}}, \gamma_{\mathrm{e}}+\gamma_{\mathrm{i}}$ & $\mathrm{k}, \sigma, \alpha, \sigma_{\mathrm{H}}, \gamma_{\mathrm{e}}+\gamma_{\mathrm{i}}$ \\
\hline $\begin{array}{c}\text { P/R scaling } \\
\text { (free to adjust) }\end{array}$ & $\begin{array}{c}\mathrm{b}^{2} \Delta_{\mathrm{p}} / \mathrm{L} \\
\left(\Delta_{\mathrm{p}}, \mathrm{b}, \mathrm{B}\right)\end{array}$ & $\begin{array}{l}\text { constant } \\
\text { (B) }\end{array}$ & $\begin{array}{c}b^{2} \Delta_{p} / L \\
(c V, b, B)\end{array}$ \\
\hline
\end{tabular}

Table 2. Some Additional Constraints and Resulting Scalings for No Neutral Model

\begin{tabular}{|c|c|c|}
\hline Case & Additional Constraints & Resulting P/R Scaling \\
\hline (a) & $\begin{array}{c}\mathrm{b}=\text { constant } \\
\Delta_{\mathrm{p}} / \mathrm{L}=\text { constant }\end{array}$ & constant \\
\hline (b) & $\begin{array}{c}\mathrm{b} \Delta_{\mathrm{p}} / \mathrm{L}=\text { constant } \\
\mathrm{B} \Delta_{\mathrm{p}}=\text { constant }\end{array}$ & b \\
\hline (c) & $\begin{array}{c}\beta \propto \mathrm{P}_{\mathrm{v}} / \mathrm{B}^{2}=\text { constant } \\
\mathrm{B} \Delta_{\mathrm{p}}=\text { constant }\end{array}$ & $\alpha \gamma_{\mathrm{e}} B_{p}$ \\
\hline (d) & $\begin{array}{c}\mathrm{b}=\text { constant } \\
\mathrm{B} \Delta_{\mathrm{p}}=\text { constant }\end{array}$ & $1 / \mathrm{BL}$ \\
\hline (e) & $\begin{array}{c}b=\text { constant } \\
\beta \propto \mathrm{P}_{\mathrm{y}} / \mathrm{B}^{2}=\text { constant }\end{array}$ & $\alpha \gamma_{e} B^{2} \triangle_{p}$ \\
\hline
\end{tabular}

